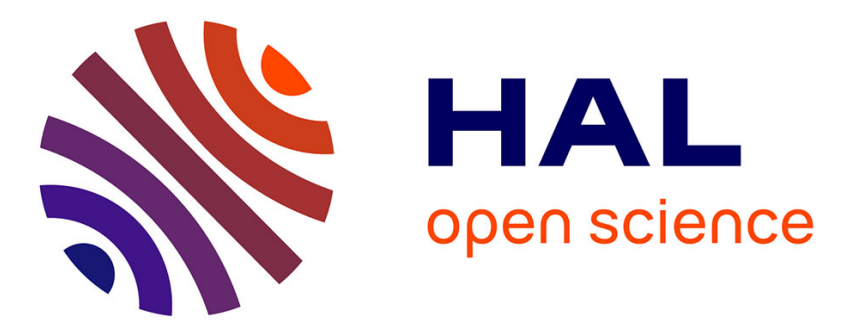

\title{
How large-scale and cyclogeostrophic barotropic instabilities favor the formation of anticyclonic vortices in the ocean
}

Gaële Perret, Thomas Dubos, Alexandre Stegner

\section{- To cite this version:}

Gaële Perret, Thomas Dubos, Alexandre Stegner. How large-scale and cyclogeostrophic barotropic instabilities favor the formation of anticyclonic vortices in the ocean. Journal of Physical Oceanography, 2011, 41 (2), pp.303-328. 10.1175/2010jpo4362.1 . hal-00838872

HAL Id: hal-00838872

https://hal-ensta-paris.archives-ouvertes.fr/hal-00838872

Submitted on 17 Nov 2021

HAL is a multi-disciplinary open access archive for the deposit and dissemination of scientific research documents, whether they are published or not. The documents may come from teaching and research institutions in France or abroad, or from public or private research centers.
L'archive ouverte pluridisciplinaire HAL, est destinée au dépôt et à la diffusion de documents scientifiques de niveau recherche, publiés ou non, émanant des établissements d'enseignement et de recherche français ou étrangers, des laboratoires publics ou privés.

\section{(c)(1)}

Distributed under a Creative Commons Attribution| 4.0 International License 


\title{
How Large-Scale and Cyclogeostrophic Barotropic Instabilities Favor the Formation of Anticyclonic Vortices in the Ocean
}

\author{
GAËLE PERRET \\ Laboratoire Ondes et Milieux Complexes, Université du Havre, Le Havre, France \\ Thomas Dubos \\ Laboratoire de Météorologie Dynamique, Ecole Polytechnique, Palaiseau, France \\ ALEXANDRE STEGNER \\ Laboratoire de Météorologie Dynamique, ENS, Paris, and UME, ENSTA Centre de l'Yvette, \\ Chemin de la Hunière, Palaiseau, France
}

(Manuscript received 11 September 2009, in final form 22 September 2010)

\begin{abstract}
Large-scale vortices, that is, eddies whose characteristic length scale is larger than the local Rossby radius of deformation $R_{d}$, are ubiquitous in the oceans, with anticyclonic vortices more prevalent than cyclonic ones. Stability or robustness properties of already formed shallow-water vortices have been investigated to explain this cyclone-anticyclone asymmetry. Here the focus is on possible asymmetries during the generation of vortices through barotropic instability of a parallel flow. The initial stage and the nonlinear stage of the instability are studied by means of linear stability analysis and direct numerical simulations of the one-layer rotating shallow-water equations, respectively. A wide variety of parallel flows are studied: isolated shears, the Bickley jet, and a family of wakes obtained by combining two shears of opposite signs.

The results show that, when the flow is characterized by finite relative isopycnal deviation, the barotropic instability favors the formation of large-scale anticyclonic eddies. The authors emphasize here that the cycloneanticyclone asymmetry of parallel flows may appear at the linear stage of the instability. This asymmetry finds its origin in the linear stability property of localized shear flows. Indeed, for both the cyclogeostrophic regime (finite Rossby number) and the frontal geostrophic regime (small Burger number), an anticyclonic shear flow has higher linear growth rates than an equivalent cyclonic shear flow. The nonlinear saturation then leads to the formation of almost axisymmetric anticyclones, while the cyclones tend to be more elongated in the shear direction.

However, although some unstable parallel flows exhibit the asymmetry at the linear stage, others exhibit such asymmetry at the nonlinear stage only. If the distance separating two shear regions is large enough, the barotropic instability develops independently in each shear, leading in the frontal and the cyclogeostrophic regime to a significant cyclone-anticyclone asymmetry at the linear stage. Conversely, if the two shear regions are close to each other, the shears tend to be coupled at the linear stage. The most unstable perturbation then resembles the sinuous mode of the Bickley jet, making no distinction between regions of cyclonic or anticyclonic vorticity. Nevertheless, when the nonlinear saturation occurs, large-scale anticyclones tend to be axisymmetric while the cyclonic structures are highly distorted and elongated along the jet meander.
\end{abstract}

\section{Introduction}

In situ measurements and general circulation models have shown that large-scale vortices, that is, eddies

Corresponding author address: Gaële Perret, Laboratoire Ondes et Milieux Complexes, 53, rue Prony, BP540, 76058 Le Havre CEDEX, France.

E-mail: perretg@univ-lehavre.fr whose characteristic length scale is larger than the local deformation radius, are ubiquitous in the oceans (Olson 1991; McWilliams 1985). A striking characteristic of these large-scale and long-lived structures is that anticyclonic vortices tend to be more prevalent than cyclonic ones. Large-scale anticyclones are frequently observed in the lee of an oceanic archipelago such as Hawaii (Mitchum 1995; Flament et al. 2001) and the Canaria (Sangrá et al. 2005) or in the vicinity of the Agulhas (Olson and Evans 
1986) and the Brazilian currents. For all of these various configurations the coastal boundary of the archipelago or a cape induces significant shear flows in the open ocean far away from the coast.

To explain the predominance of anticyclones among large-scale eddies, several studies were devoted to the specific stability of anticyclonic vortices in rotating shallow-water flows (Arai and Yamagata 1994; Stegner and Dritschel 2000; Baey and Carton 2002). Moreover, stable anticyclones tend to remain coherent within a turbulent flow (Polvani et al. 1994; Arai and Yamagata 1994; Linden et al. 1995) and they were found to be more robust to external strain perturbations than cyclonic eddies (Graves et al. 2006). Taking into account the weak beta effect, which may affect large-scale oceanic eddies, reveals that only anticyclones are weakly affected by the Rossby wave dispersion relation for a long time (Matsuura and Yamagata 1982; Nycander and Sutyrin 1992; Stegner and Zeitlin 1995, 1996).

However, very few studies investigate how the generation process may itself induce an initial selection between cyclonic or anticyclonic eddies. Most of the mesoscale oceanic vortices are formed by the unstable meanders of shear, jet, or wake flows occurring in coastal regions or in the open sea. The instability of oceanic currents has often been modeled using quasigeostrophic (QG) theory since they typically have small Rossby number. Using the QG equations, valid for small Rossby number and small isopycnal displacement, a flow and its mirror image evolve identically. Therefore, the baroclinic or the barotropic instabilities of a jet or a wake flow generate eddies of both signs having the same size or intensity. However, in the full shallow-water system, the mirror symmetry does not hold. For instance, when the relative surface deviation becomes finite, a cyclone and an anticyclone with equal and opposite relative vorticity produce PV anomalies of different magnitudes. Therefore, the departure from the QG regime (finite isopycnal displacement and/or finite Rossby number) may induce a specific cyclone-anticyclone asymmetry.

A study (Poulin and Flierl 2003) has investigated the linear stability of a Bickley jet and its nonlinear evolution in the framework of rotating shallow-water (RSW) equations. At the linear stage, the most unstable mode is sinuous and the jet meanders with no distinction between the cyclonic and the anticyclonic side. However, beyond the quasigeostrophic regime, when the Rossby number becomes finite and induces large isopycnal displacements due to the cyclogeostrophic balance, the nonlinear evolution of the instability leads to asymmetric eddy formation: the cyclones tend to be elongated and stretched in comparison with almost circular anticyclones. Other recent papers have investigated, by means of laboratory experiments (Perret et al. 2006b) or stability analysis (Perret et al. 2006a), the large-scale wake of circular islands. For the frontal geostrophic regime, that is, small Rossby number and finite isopycnal displacement (Cushman-Roisin 1986; CushmanRoisin and Tang 1990), a significant asymmetry occurs in the wake between cyclonic and anticyclonic vortices. For some extreme cases, coherent cyclones do not emerge at all, and only an anticyclonic vortex street appears several diameters behind the circular island. This asymmetry was first explained by the linear stability analysis of parallel wake flows in the framework of RSW equations (Perret et al. 2006a). Indeed, in the frontal regime, the most unstable mode is fully localized in the anticyclonic shear region. Hence, the anticyclonic perturbations, leading to large-scale anticyclones, have the fastest growth rates. $\mathrm{Di}$ rect numerical simulations show that the nonlinearities exacerbate the dominance of the anticyclonic mode linearly selected. Here again, when they are formed, the cyclones tend to be stretched and elongated in comparison with the large circular anticyclones. Anticyclonic predominance was also found in a fully stratified simulation, using the Regional Ocean Modeling System (ROMS), when the island size becomes larger than the local deformation radius (Dong et al. 2007). Hence, both linear and nonlinear instability of parallel jets or wake flows may induce an asymmetric eddy formation.

The main goal of the present work is to understand how the barotropic instability of various parallel flows (shears, jets, and wakes) may favor the formation of large-scale anticyclonic eddies. In which dynamical regimes will the anticyclonic predominance become significant? Why does this asymmetry appear at the linear stage for a parallel wake flow (Perret et al. 2006a) and at the nonlinear stage for the Bickley jet (Poulin and Flierl 2003)?

In section 2 we discuss the dynamical parameters governing the RSW model and the numerical methods used for the linear stability analysis and to compute the full nonlinear equations. As a first step, in section 3 we study the linear and the nonlinear stability of localized cyclonic and anticyclonic shear flows. In section 4 , the stability of the Bickley jet is investigated from the quasigeostrophic regime to the frontal geostrophic regime. In section 5, various wake profiles are constructed as a combination of two localized shears. At this stage we introduce a new dynamical parameter to quantify the distance between the two shears and analyze its impact on the stability of the parallel wake flows. We then discuss and summarize the results in section 6 .

\section{Model}

\section{a. Rotating shallow-water equations and dynamical regimes}

As a first approximation of surface oceanic flows, we use a reduced gravity model, also known as a 1/2-layer 
model, assuming a thin upper layer above a motionless deep bottom layer. According to Cushman-Roisin (1986), if the ratio of the upper-layer depth to the total depth of the ocean is smaller than $\min (1, \mathrm{Bu})$, the upper layer will not be affected by the bottom-layer dynamics. Hence, the upper-layer motion follows the RSW equations. We use below the dimensionless form of the RSW model on the $f$ plane,

$$
\operatorname{Ro}\left(\frac{\partial \mathbf{V}}{\partial t}+(\mathbf{V} \cdot \nabla) \mathbf{V}\right)+\mathbf{n} \times \mathbf{V}=-\frac{\mathrm{Bu}}{\mathrm{Ro}} \nabla \eta+\frac{\mathrm{Ro}}{\mathrm{Re}} \nabla^{2} \mathbf{V}
$$

and

$$
\left(\frac{\partial \eta}{\partial t}+\mathbf{V} \cdot \nabla \eta\right)+(1+\eta) \boldsymbol{\nabla} \cdot \mathbf{V}=0
$$

where $\mathbf{n}$ is the upward-pointing unit vector, space is scaled by a characteristic length scale $L, \mathbf{V}=(u, v)$ is the horizontal velocity scaled by the typical velocity $V_{0}, t$ is the time scaled by the advective time scale $L / V_{0}$, and $\eta$ is the surface deviation scaled by the unperturbed layer depth $h_{0}$. Notice that this scaling is slightly different from the one used by Perret et al. (2006a). In particular, $\eta$ is scaled here by the total unperturbed depth $h_{0}$ and not by its typical fluctuation $\Delta h_{0}$, and is not necessarily of order 1 . We define the Rossby number Ro, the relative surface deviation parameter $\lambda$, the Burger number $\mathrm{Bu}$, and the Reynolds number Re as follows:

$$
\mathrm{Ro}=\frac{V_{0}}{f L}, \quad \lambda=\frac{\mathrm{Ro}}{\mathrm{Bu}}, \quad \mathrm{Bu}=\left(\frac{R_{d}}{L}\right)^{2}, \quad \mathrm{Re}=\frac{V_{0} L}{\nu}
$$

with $R_{d}=\sqrt{g^{\prime} h_{0}} / f$ the deformation radius, $f=2 \Omega$ the Coriolis parameter, $g^{\prime}$ the reduced gravity acceleration, and $\nu$ the kinematic viscosity.

When the Rossby number is small, the geostrophic balance is satisfied at leading order, imposing the surface deviation to balance the Coriolis term in Eq. (1). Thus, the dominant balance imposes $\eta=O(\lambda)$, meaning that the amplitude of the (dimensional) surface deviation scales like $L V_{0} f / g$.

At small Rossby number, one distinguishes two dynamical regimes depending on the value of the Burger number. The classical quasigeostrophic regime (Pedlosky $1987)$ is defined when $\mathrm{Bu}=O(1)$ or equivalently $\lambda=$ $O($ Ro $) \ll 1$, leading to relative small surface deviation. The QG model [Eq. (3)] corresponds to the quasigeostrophic regime asymptotic expansion truncated at the first order in Rossby number,

$$
\frac{\partial}{\partial t}\left(\Delta \psi-\mathrm{Bu}^{-1} \psi\right)-J(\psi, \Delta \psi)=0
$$

where

$$
\psi=\eta / \lambda=O(1)
$$

In this limit the mirror symmetry $\mathbf{y} \rightarrow-\mathbf{y}$ and $\psi \rightarrow-\psi$ holds at the leading order, meaning that the dynamic of cyclonic and anticyclonic structures are interchangeable. Even if the Rossby number is not asymptotically small in this study $(\mathrm{Ro}=0.1)$, cyclones and anticyclones are expected to follow roughly the same evolution when $\mathrm{Bu}=O(1)$. The second regime corresponds to the frontal regime and is defined by $\mathrm{Bu}=O(\mathrm{Ro}) \ll 1$. In this regime, the flow is expected to follow at leading order the frontal geostrophic (FG) asymptotic model (Cushman-Roisin 1986; Cushman-Roisin and Tang 1990). The latter, Eq. (4), is derived according to an asymptotic expansion $(\lambda \simeq 1$, Ro $\ll 1)$ truncated at second order in Rossby number. As a result, the frontal geostrophic time scale is $O(\mathrm{Ro})$ slower than the quasigeostrophic time scale:

$$
\frac{\partial \eta}{\partial t^{\prime}}-J\left(\eta,(1+\eta) \Delta \eta+\frac{1}{2}(\nabla \eta)^{2}\right)=0
$$

in which $t^{\prime}=$ Rot. In this model the surface deviations are of order unity, the mirror symmetry is broken, and the evolution of cyclonic and anticyclonic structures may differ strongly.

Notice that we distinguish between the dynamical regimes and the balanced asymptotic models. Unlike the RSW equations that can induce fast wave motions, the standard QG and FG models correspond to balanced equations where inertia-gravity waves are filtered out. The simplicity of the balanced models allows the derivation of relatively simple stability criteria (see appendix A). In both regimes, it is found that a parallel flow that obeys the geostrophic balance

$$
\bar{U}(y)=-\frac{1}{\lambda} \frac{\partial \bar{\eta}}{\partial y}
$$

will be linearly stable if the corresponding potential vorticity, $q=(\operatorname{Ro} \nabla \times \mathbf{V}+1) /(1+\eta)$, is monotonic. However, these generalized Rayleigh criteria do not induce any asymmetry in the stability properties of parallel flows (shears, jets, and wakes) and a complete stability analysis is therefore needed to understand the cyclone-anticyclone asymmetry of large-scale flows.

When the Rossby number becomes finite $(\mathrm{Ro} \simeq 1)$, the balance between the velocity and the pressure field (i.e., the surface deviation for the RSW equations) is not trivial. There is no simple balance relation valid at any time. We cannot construct asymptotic expansions 

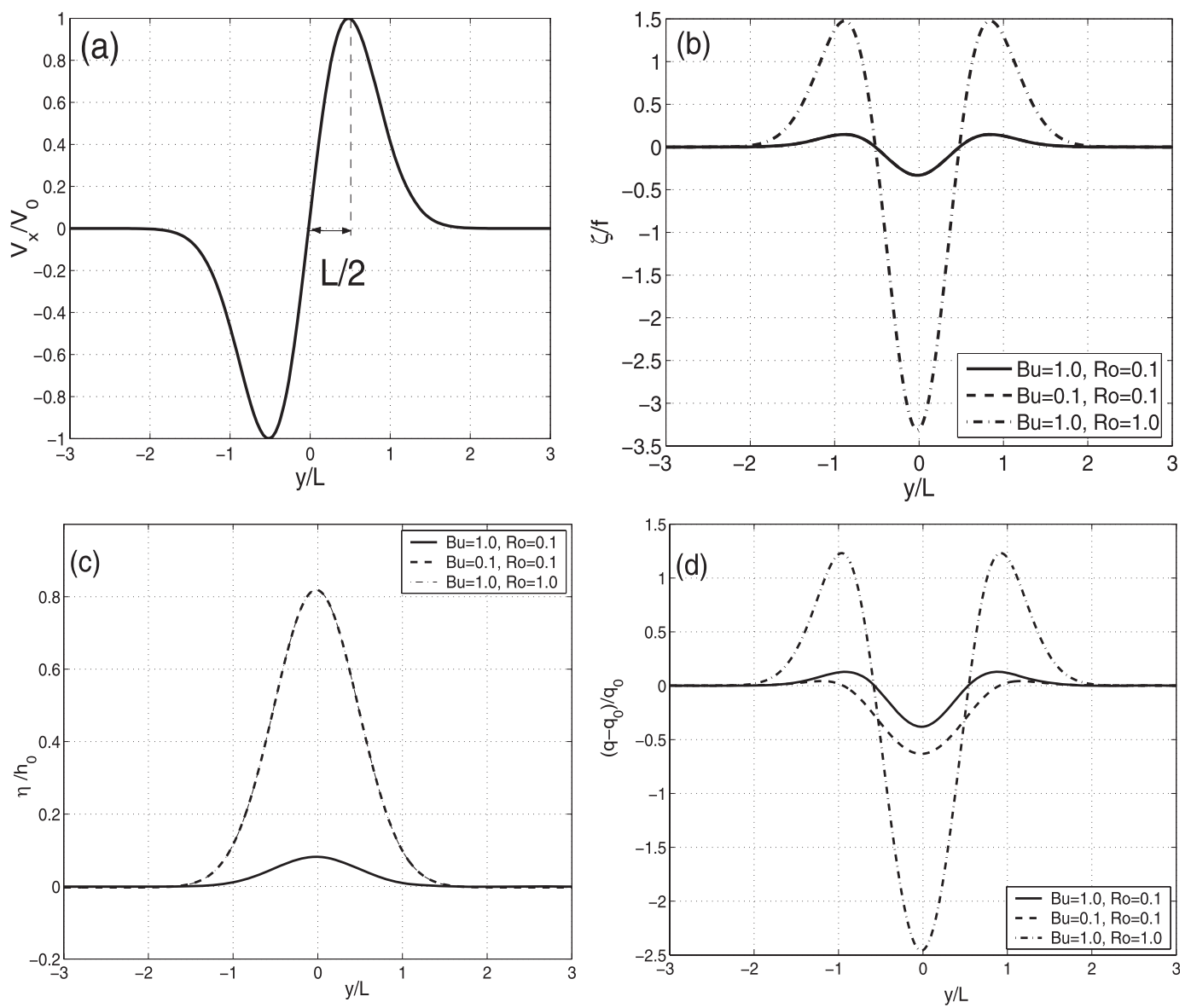

FIG. 1. Anticyclonic shear basic state profiles: (a) velocity, (b) vorticity, (c) relative surface deviation, and (d) PV for QG regime (solid line), frontal regime (dashed line), and cyclogeostrophic regime (dotted-dashed line). [Note that in (b) QG and frontal vorticity and in (c) frontal and cyclogeostrophic relative surface deviation are superimposed.]

and balanced models valid for finite Rossby numbers. Nevertheless, circular and steady vortices follow the nonlinear cyclogeostrophic balance and steady parallel flows follow the geostrophic balance. Hence, the initial steady state of the shears, jets, and wakes flows that we studied in the cyclogeostrophic regime $(\mathrm{Ro} \simeq 1, \mathrm{Bu} \simeq 1)$ satisfies the previous scaling for the surface deviation $\eta \simeq \lambda$.

For a given parallel velocity profile $\bar{U}(y)$ (Fig. 1a), the relative surface deviation in geostrophic balance with $\bar{U}(y)$ (Fig. 1c) increases in amplitude when the dynamical regime is varied from a quasigeostrophic $[\lambda=$ $O(\mathrm{Ro}) \ll 1]$ to a frontal regime $[\mathrm{Bu}=O(\mathrm{Ro}) \ll 1]$ or a cyclogeostrophic regime $(\mathrm{Bu} \simeq 1$ and $\mathrm{Ro} \simeq 1)$. When the width $L$ of the parallel flow becomes larger than the deformation radius or the typical flow velocity $\mathbf{V}_{0}$ reaches $f L$, the relative surface deviation (Fig. 1c) becomes finite $(\lambda \simeq 1)$. Unlike the latter, the potential vorticity fluctuations (Fig. 1d) are controlled by two independent parameters $\lambda$ and Ro. Hence, an initial parallel flow satisfying the frontal regime or the cyclogeostrophic regime will correspond to two distinct initial states. Cyclone-anticyclone asymmetry is thus characterized throughout the study using potential vorticity fluctuations.

To study the flow stability from the QG regime to the $\mathrm{FG}$ regime, the Burger number is varied from $\mathrm{Bu}=1$ to $\mathrm{Bu}=0.1$ while the Rossby number is kept fixed at Ro $=$ 0.1 . To study the stability properties from the QG to the cyclogeostrophic regime, the Rossby number is varied from $\mathrm{Ro}=0.1$ to $\mathrm{Ro}=1$ while the Burger number is kept fixed at $\mathrm{Bu}=1$. Hence, in both cases, the value of the relative deviation parameter $\lambda$ is varied from $\lambda=0.1$ to $\lambda=1.0$ to get a significant deviation from the QG dynamics.

\section{b. Numerical resolution}

For each type of parallel flow we perform a temporal stability analysis and compute the nonlinear evolution of 
the perturbed flow in the framework of the RSW equations. The numerical methods are identical to those used in Perret et al. (2006a). They are briefly presented below for completeness and clarity.

\section{1) LINEAR STABILITY ANALYSIS}

To perform the temporal stability analysis, the shallowwater equations are linearized around a parallel basic state in geostrophic balance $(\bar{U}(y), \bar{\eta}(y))$. Assuming that the instability mechanism is inviscid, we consider the RSW equations without viscous terms. The perturbation field is expanded in normal modes:

$(\tilde{u}(x, y, t), \tilde{v}(x, y, t), \tilde{\eta}(x, y, t))=(\widehat{u(y)}, \widehat{v(y)}, \widehat{\eta(y)}) e^{i(k x-\omega t)}$

with $\omega=\omega_{r}+i \sigma$ the complex eigenfrequency and $k$ the real wavenumber. We then obtain the eigenvalue problem:

$$
\begin{aligned}
& \mathcal{L}\left(\begin{array}{c}
\hat{u} \\
\hat{v} \\
\hat{\eta}
\end{array}\right)=\omega\left(\begin{array}{l}
\hat{u} \\
\hat{v} \\
\hat{\eta}
\end{array}\right) \text { with } \\
& \mathcal{L}=\left(\begin{array}{ccc}
-k \lambda^{-1} \frac{\partial \bar{\eta}}{\partial y} & i\left(\lambda^{-1} \frac{\partial^{2} \bar{\eta}}{\partial y^{2}}+\mathrm{Ro}^{-1}\right) & \mathrm{Ro}^{-1} \lambda^{-1} k \\
-i \mathrm{Ro}^{-1} & -k \lambda^{-1} \frac{\partial \bar{\eta}}{\partial y} & i \mathrm{Ro}^{-1} \lambda^{-1} \frac{\partial}{\partial y} \\
(1+\bar{\eta}) k & -i\left(\frac{\partial \bar{\eta}}{\partial y}+(1+\bar{\eta}) \frac{\partial}{\partial y}\right) & -k \frac{\partial \bar{\eta}}{\partial y}
\end{array}\right) .
\end{aligned}
$$

An approximation $\mathcal{L}_{N}$ of the matrix of the linear operator $\mathcal{L}$ is computed in a spectral basis with periodic boundary conditions. With $N$ Fourier modes, $\mathcal{L}_{N}$ is a $3 N \times 3 N$ matrix, which we diagonalize using the zgeev function of LAPACK linear algebra package (Anderson et al. 1999). This provides the full eigenvalue spectrum of $\mathcal{L}_{N}$. Among the $3 N$ complex eigenvalues, only 0,1 , or 2 are found to have a positive real part $\sigma_{N}$.

The convergence of solutions is obtained for a resolution varying between $N=256$ and $N=2048$, depending of the basic state and the dynamical regime. Starting with $N=256$, we double $N$ and monitor the relative difference between successive approximations of $\sigma$. Specifically, we compute

$$
\varepsilon_{N}=\frac{\sum_{j}\left(\sigma_{2 N}\left(k_{j}\right)-\sigma_{N}\left(k_{j}\right)\right)^{2}}{\sum_{j} \sigma_{2 N}\left(k_{j}\right)^{2}},
$$

where the $k_{j}$ are a number of wave vectors spanning an interval $\left[k_{\min }, k_{\max }\right]$ of interest. As in Perret et al. (2006a), we stop doubling $N$ when $\varepsilon_{N}$ becomes $O\left(10^{-4}\right)$.

\section{2) NONLINEAR EVOLUTION}

The nonlinear evolution of the instability is studied by computing the nonlinear evolution of the perturbed parallel flows. The rotating shallow-water equations are discretized in space with a pseudospectral method and in time with a second-order leapfrog scheme. To lower the computation time, the domain is reduced to two wavelengths of the most unstable mode in the streamwise direction, as determined from the linear stability analysis. The resolution, in the spanwise direction, is 256 and the boundary conditions are periodic. The basic state is parallel with the same velocity profile extended to the entire domain. The Reynolds number is fixed at $\mathrm{Re}=$ 9000 to reduce viscous effects. When a numerical simulation is initialized with a parallel basic velocity profile, without external perturbations, the flow stays parallel and the velocity diffuses slowly. The basic state velocity is initially perturbed with a random perturbation field whose fluctuation level is about $0.1 \%$ times the velocity amplitude. Furthermore, the initial surface deviation is in geostrophic balance with the initial velocity deviation so as to avoid gravity wave emission.

\section{Localized shear flow}

\section{a. Basic flow}

The hyperbolic tangent velocity profile, $U(y)=$ $V_{0} \tanh y / L$, was often used as a generic flow to study the stability of a single two-dimensional shear flow (Drazin and Reid 1981; Johnson 1963). However, in the framework of the RSW equations, the stability of such shear flow may strongly depend on the domain size. Indeed, due to the geostrophic balance, the constant velocities values away from the shear zone control the surface deviation in the central shear zone. If the domain size is too large, outcropping (vanishing layer depth) may occur. To avoid this undesirable influence of the domain size, the velocity field should vanish far away from the shear. Therefore, instead of using a standard hyperbolic tangent shear flow, we study, in this section, the stability of a localized shear flow defined in a dimensionless form by

$$
\bar{U}(y)=\frac{U(y)}{V_{0}}=c y e^{-2 y^{2}} ; \quad \bar{\eta}(y)=\frac{\eta(y)}{h_{0}}=c \frac{\lambda}{4} e^{-2 y^{2}}
$$

with $y \in[-5,5]$, where $c=2 e^{1 / 2}$. With this normalization coefficient, the localized shear flow is anticyclonic and extremum velocity values $U / V_{0}=1$ and $U / V_{0}=-1$ occur at $y=1 / 2$ and $y=-1 / 2$. In this case, the minimal vorticity value is negative and centered in $y=0$, whereas two positive vorticity peaks (having weaker amplitudes) 


\section{anticyclone}
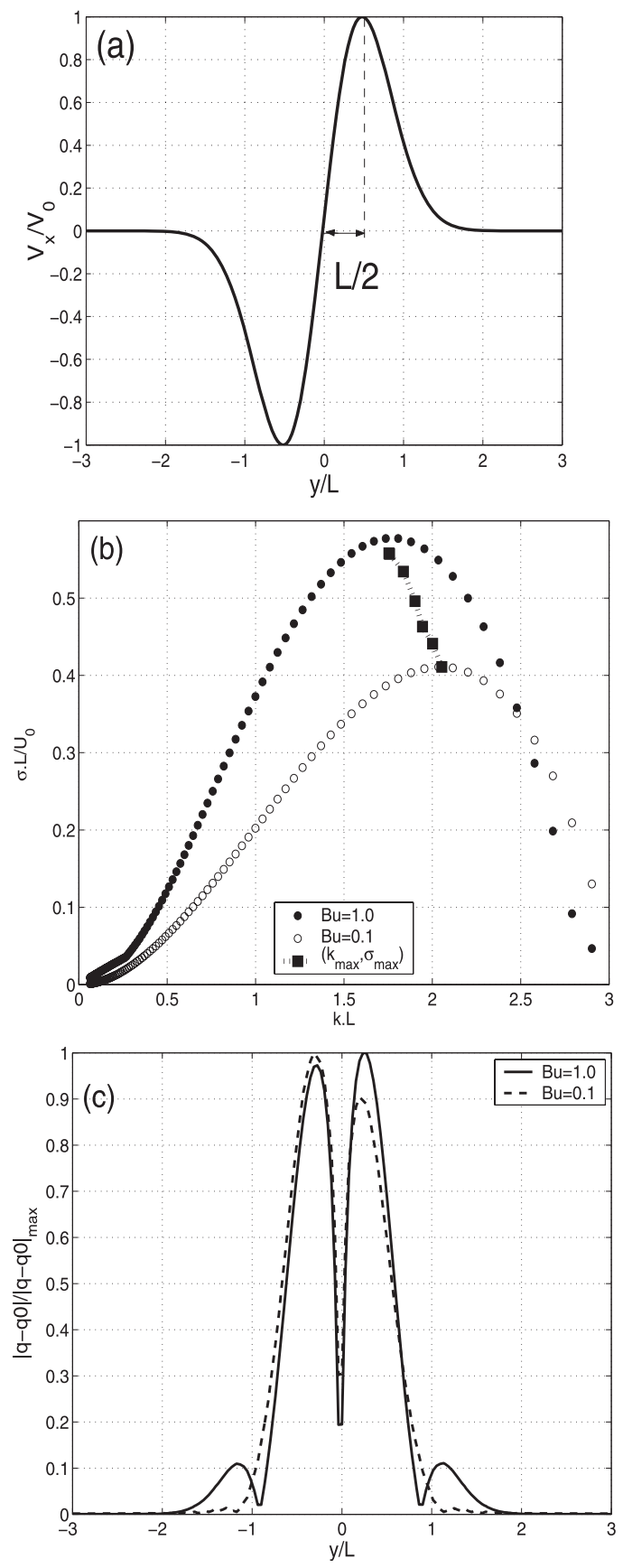

cyclone
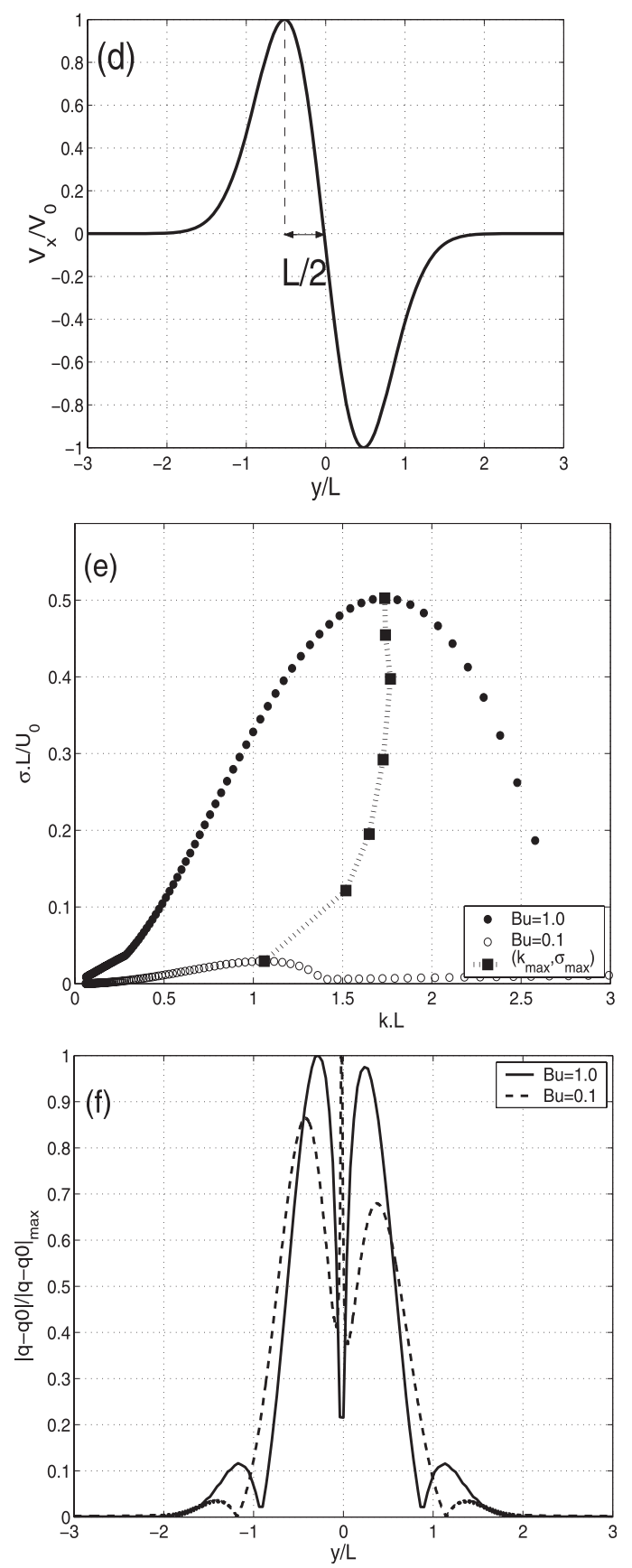

FIG. 2. Stability properties of the anticyclonic and cyclonic localized shear flow (a),(d) for Ro $=0.1$ and various Burger numbers. (b),(e) The unstable growth rates, calculated both for the QG (filled dots) and the frontal regime (open dots). (c),(f) The PV anomaly $\left[\left(q-q_{0}\right)\right.$, where $q_{0}=f / h_{0}$ is the mean PV, normalized by $\left.\left(q-q_{0}\right)_{\max }\right]$ of the most unstable modes are given.

correspond to the lateral shears (Fig. 1b). A cyclonic shear is obtained when $c=-2 e^{1 / 2}$. In appendix $\mathrm{B}$, we show that these lateral shears have little influence on the growth rate of the most unstable mode. b. Linear instability in the $Q G, F G$, and cyclogeostrophic regimes

Figure 2 compares the unstable growth rates $\sigma_{A}$ and $\sigma_{C}$ (Figs. 2b,e) and the leading normal modes (Figs. 2c,f) 
of an anticyclonic (Fig. 2a) and a cyclonic (Fig. 2d) localized shear flow. A resolution of $N=256$ was shown to be sufficient to get convergence of the growth rates $\left(\varepsilon_{256}=1.6 \times 10^{-4}\right)$. However, it turns out that the most unstable cyclonic normal mode presents strong discontinuities in a frontal regime (Fig. 2f, dashed curve, sharp pike near $y=0$ ), and it was necessary to increase the number of collocation points for these computations, up to $N=2048$.

In the quasigeostrophic regime, corresponding here to $\lambda=0.1$ and Ro $=0.1$, the growth rates and the wavelengths of the most unstable perturbations in the cyclonic and anticyclonic shear are very close. The slight differences in the growth rate are due to small ageostrophic effects since the Rossby number, Ro $=0.1$, in our calculation is small but finite. To confirm this, we have also performed this stability analysis with QG dynamics, that is, by linearizing the QG asymptotic Eq. (3) around the same velocity profile (not shown). As expected from the mirror symmetry of the QG equations, the cyclonic and the anticyclonic localized shear flows have the same growth rates. Furthermore, the QG growth rates are close to the shallow-water growth rates at $\mathrm{Ro}=$ 0.1 , indicating that the slight difference between $\sigma_{A}$ and $\sigma_{C}$ results, indeed, from the small but finite Ro.

In a frontal regime, corresponding here to $\mathrm{Bu}=0.1$ and Ro $=0.1$, the dimensionless growth rate of the cyclonic and the anticyclonic shears differ greatly. While the growth rate in the anticyclonic shear decreases by only $30 \%$ from its quasigeostrophic value, the unstable growth rate within the cyclonic shear strongly decreases. For the latter the maximum growth rate in the FG regime is only $6 \%$ of the maximum growth rate in the $\mathrm{QG}$ regime (Fig. 2e).

We should note, however, that in the FG regime the growth rates of both shears decrease a finite amount. The growth rate associated to barotropic instability generally decreases when the Burger number decreases. This was already the case within the standard QG model if we reduce the Burger number in Eq. (3). This largescale stabilization was also quantified in the RSW model for the Bickley jets (Poulin and Flierl 2003) or isolated eddies (Stegner and Dritschel 2000) in the frontal regime. Similar tendency were also found for specific wake flows (Perret et al. 2006a). This general stabilization of large-scale barotropic flows is confirmed by the multiplescale asymptotic expansion of the one-layer RSW equations (Cushman-Roisin 1986; Stegner and Zeitlin 1996). Indeed, the typical time scale $\left(\mathrm{Ro}^{2} f\right)^{-1}$ for the asymptotic FG model is one order of magnitude larger than the typical time scale $(\operatorname{Ro} f)^{-1}$ of the QG model. In other words, for the same velocity amplitude (same Ro) largescale flows (small $\mathrm{Bu}$ ) of both signs (with cyclonic or anticyclonic vorticity) evolve more slowly than quasigeostrophic flows (finite or large $\mathrm{Bu}$ ). This is of course not the case for a two-layer configuration where the baroclinic instability could play an important role.

In the cyclogeostrophic regime, corresponding here to $\mathrm{Bu}=1$ and $\mathrm{Ro} \simeq 1$, we found a more pronounced asymmetry between the unstable growth rate of the cyclonic and the anticyclonic shears (Fig. 3). In contrast with the FG regime, the growth rates of the localized anticyclonic shear do not decrease and remain almost constant while the Rossby number increases. On the other hand, as for the FG regime, a strong stabilization of the cyclonic shear occurs in the cyclogeostrophic regime. The maximum normalized growth rate when $\mathrm{Ro}=1$ is only $10 \%$ of the maximum normalized growth rate in the $\mathrm{QG}$ regime $(\mathrm{Ro}=0.1$ and $\mathrm{Bu}=1)$.

For an easier comparison with oceanic observations, we compute the $e$-folding time $1 / \sigma$ of the unstable perturbations, scaled here by the local day $T_{0}=4 \pi / f$, as a function of the relative deviation parameter $\lambda$ (Fig. 4). The $e$-folding time is mainly controlled by the local turnover time $L / V_{0}$; hence, the cyclogeostrophic shears (large $V_{0}$ or small $L$ ) tend to have much smaller $e$-folding time than the FG shears (large $L$ or small $V_{0}$ ). Nevertheless, in all the cases, when the isopycnal displacement become finite $(\lambda=1)$ the $e$-folding time of the perturbations will be one order of magnitude higher for the localized cyclonic shear than for the anticyclonic one. Hence, the cyclone-anticyclone asymmetry in the linear growth rates is mainly controlled by the relative elevation parameter $\lambda$. Both frontal regime and cyclogeostrophic regimes stabilize the cyclonic barotropic shear and, therefore, favor the development of unstable perturbations in the anticyclonic shear. In all cases, the leading unstable mode extends throughout the whole shear zone (Figs. 2c,f and 3b,d) and its shape is weakly affected by the deformation radius variations or the Rossby number values.

For the incompressible two-dimensional shear instability (Drazin and Reid 1981), the unstable wavelength is controlled by the width of the shear layer $L$. For a barotropic shear in the RSW model, the unstable wavelength could be controlled by at least two horizontal scales: $L$ and the deformation radius $R_{d}$. We plot in Fig. 5a (Fig. 5b) the most unstable wavelength normalized by $L\left(R_{d}\right){ }^{1}$ According to Fig. 5 a, the unstable

\footnotetext{
${ }^{1}$ One should note that the two normalizations are linked. Thus, for fixed Burger number, $\mathrm{Bu}=1$ and $L=R_{d}$, the evolution of the most unstable wavelength is the same for the two normalizations. For fixed Rossby number, the most unstable wavelength normalized by $R_{d}$ increases with increasing $\lambda=\mathrm{Ro} L^{2} / R_{d}^{2}$ since $2 \pi /\left(k_{\max } R_{d}\right)=$ $\left[2 \pi /\left(k_{\max } L\right)\right] L / R_{d}$.
} 


\section{anticyclone}
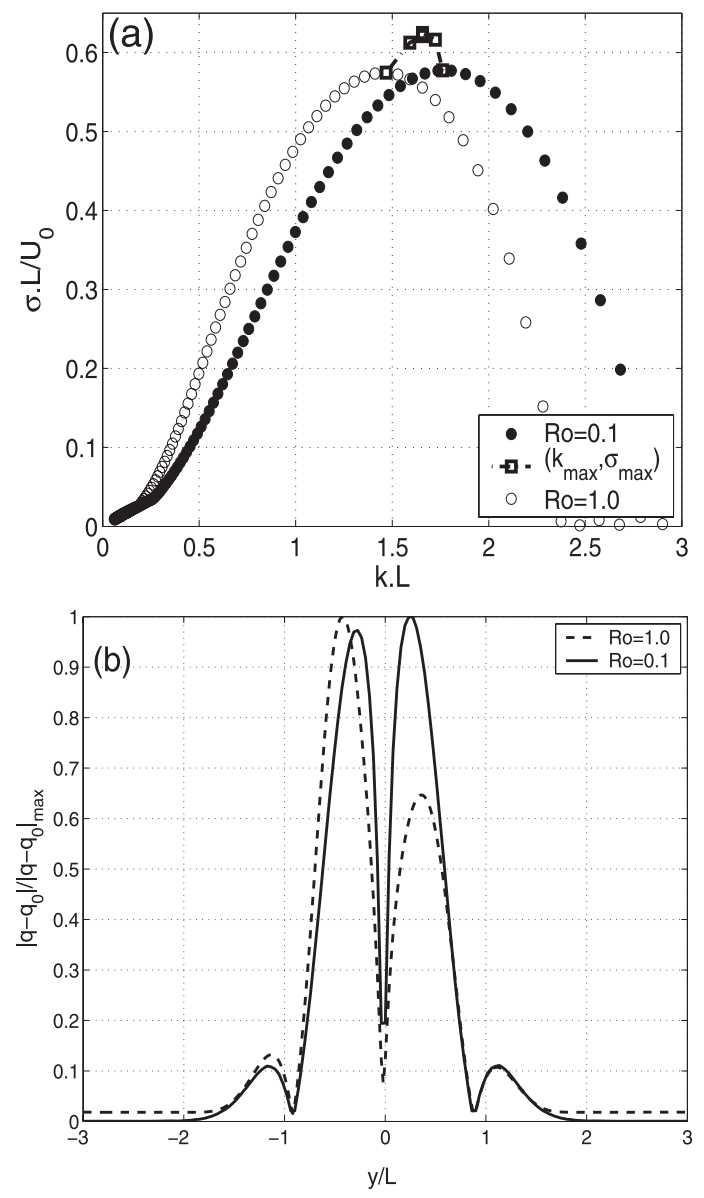

cyclone
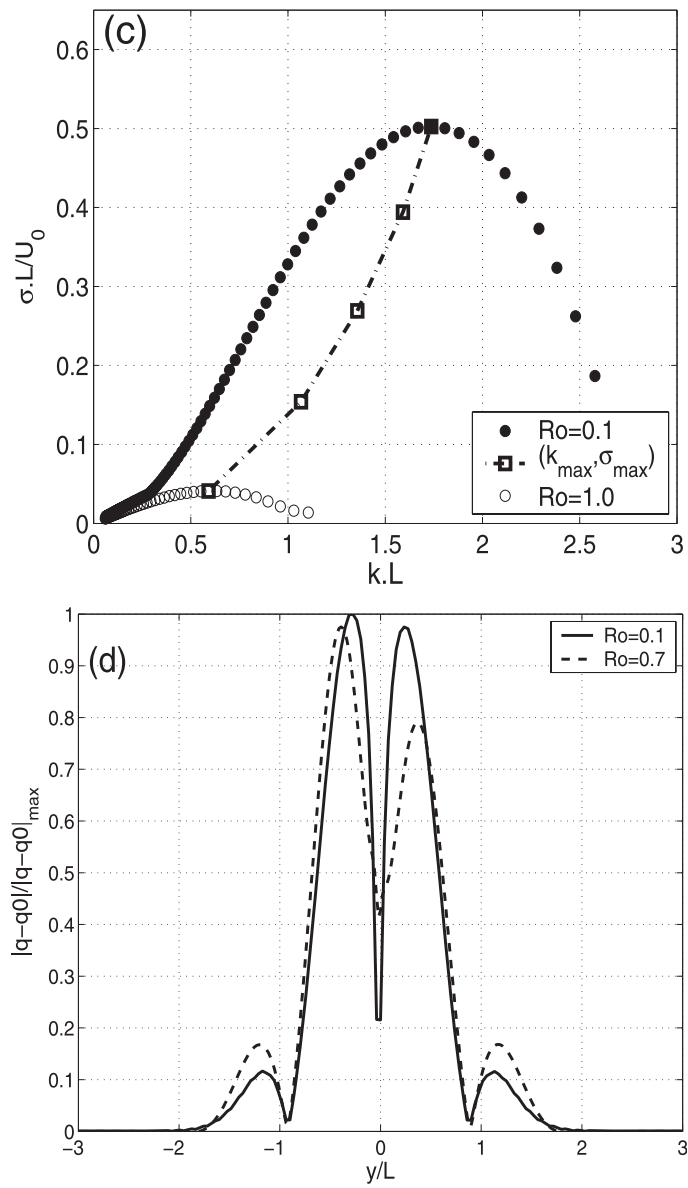

FIG. 3. Stability properties of the anticyclonic and cyclonic localized shear flow for $\mathrm{Bu}=1.0$ and various Rossby numbers. (a),(c) The unstable growth rates, calculated both for the QG (filled dots) and the cyclogeostrophic parameters (open dots). (b),(d) The PV anomaly of the most unstable modes.

wavelength of the anticyclonic shear is proportional to $L$ and is weakly affected by the variation of the deformation radius or the Rossby number while the unstable wavelength of the cyclonic shear increases significantly for large isopycnal displacement. Hence, the unstable modes tend to be more elongated in cyclonic shears than anticyclonic ones. According to Fig. 5b, the most unstable wavelength is always larger than the deformation radius, and this ratio increases from $2 \pi / k=3.5 R_{d}$ for an anticyclonic shear in the quasigeostrophic or the cyclogeostrophic regime up to $2 \pi / k \simeq 10 R_{d}$ in the frontal regime.

\section{c. Nonlinear evolution}

The nonlinear evolution of the instability and the vortex formation are studied by direct numerical simulations. The nonlinear evolution of localized shear flows are computed in the quasigeostrophic regime (Fig. 6), the frontal regime (Fig. 7), and the cyclogeostrophic regime (Fig. 8). In agreement with the linear analysis and as expected for the quasigeostrophic regime $(\mathrm{Ro}=0.1$, $\mathrm{Bu}=1$ ), the nonlinear saturation of the shear instability is very similar for cyclonic and anticyclonic shear. $\mathrm{Cy}$ clones and anticyclones are slightly out of phase but have similar shape and strength. The distance between two emerging vortices $4 L$ is very close to the most unstable wavelength $2 \pi / k=3.5 \mathrm{~L}$.

In a frontal regime (Fig. 7) the growth of the perturbations differs significantly between the cyclonic and the anticyclonic shear. Vortices appear in the anticyclonic shear at $t / T_{0}=20$ whereas the emergence of cyclonic vortices only occurs at $t / T_{0}=200 \sim 250$, where $T_{0}=4 \pi / f$ is about one day. Moreover, once they are formed within the unstable shear, cyclonic eddies tend to have a larger extension along the shear than anticyclonic ones as expected from the linear stability analysis. Indeed, in the 


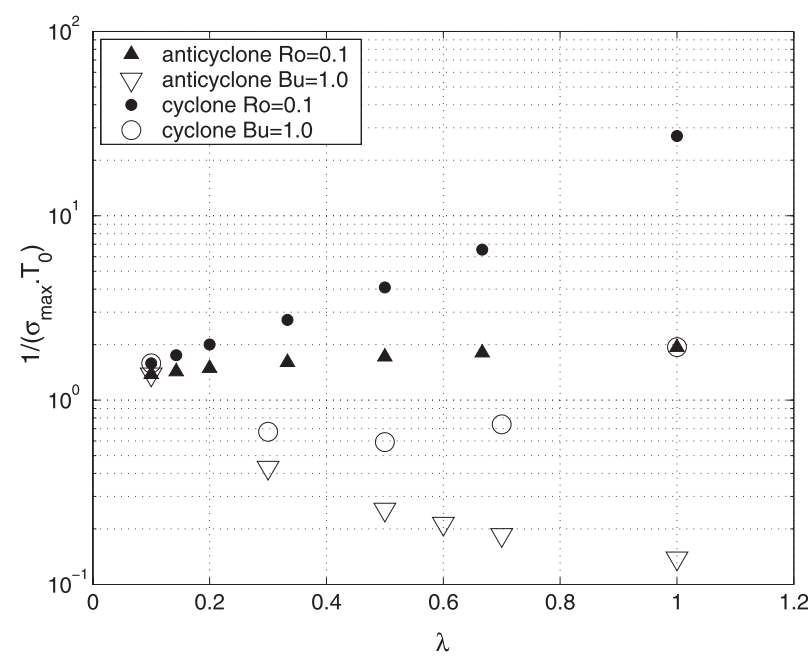

FIG. 4. Evolution of the $e$-folding time scaled by the local day for anticyclonic (filled and empty triangles) and cyclonic (filled and empty circles) shear as a function of the surface deviation $\lambda$. Filled symbols correspond to a Rossby number fixed at 0.1 and various Burger numbers, whereas open symbols correspond to a Burger number fixed at 1.0 and various Rossby numbers.

frontal regime, according to Figs. 2b,e the wavelength of the most unstable cyclonic mode is twice longer than the anticyclonic one. An axisymmetrization process generally occurs during the nonlinear saturation, but the coherent structures remain elongated in the cyclonic shear while axisymmetric eddies are formed in the anticyclonic shear (Fig. 7). According to previous studies (Arai and Yamagata 1994; Graves et al. 2006), the axisymmetrization process was found to be much weaker for elliptical cyclones beyond the QG regime when the isopycnal deviation becomes finite. The mean cyclonic shear may amplify this tendency.

In the cyclogeostrophic regime (Fig. 8), the perturbations grow faster in the anticyclonic shear, in agreement with the linear stability analysis (Fig. 3). Here again, the nonlinear evolution of the flow increases the cyclone-anticyclone asymmetry, leading to strongly elongated cyclones (Fig. 8, $t=11 T_{0}$ ) that split (Fig. 8, $t=15 T_{0}$ ) into smaller circular eddies. This significant asymmetry occurs when the relative vorticity is $O(\zeta / f \simeq 1)$, even if the global Rossby number is moderate $(\mathrm{Ro}=0.3)$. We restrict our nonlinear simulations to moderate Rossby numbers so as to avoid negative values of the potential vorticity. Indeed, for negative PV oceanic shear flow may become inertially unstable (Johnson 1963; Yanase et al. 1993; Potylitsin and Peltier 1999), and small-scale ageostrophic perturbations will grow quickly if the stratification and the dissipation are not too strong (Stegner et al. 2005; Teinturier et al. 2010). In such cases, the rotating shallow-water formulation that filters out these small-scale and three-dimensional motions could lead to unrealistic results.

Moreover, if we analyze the vorticity field during the nonlinear stage of the instability, we can detect some differences between the cyclonic and the anticyclonic structures (Fig. 9). Indeed, when the isopycnal deviations become finite, the vorticity field seems to be strongly perturbed in the core of cyclonic eddies while anticyclonic eddies remain more coherent and circular. This could be a signature of the cyclonic sensitivity to external strain perturbations, induced by the mean shear and the neighboring vortices, as suggested by the study of Graves et al. (2006).
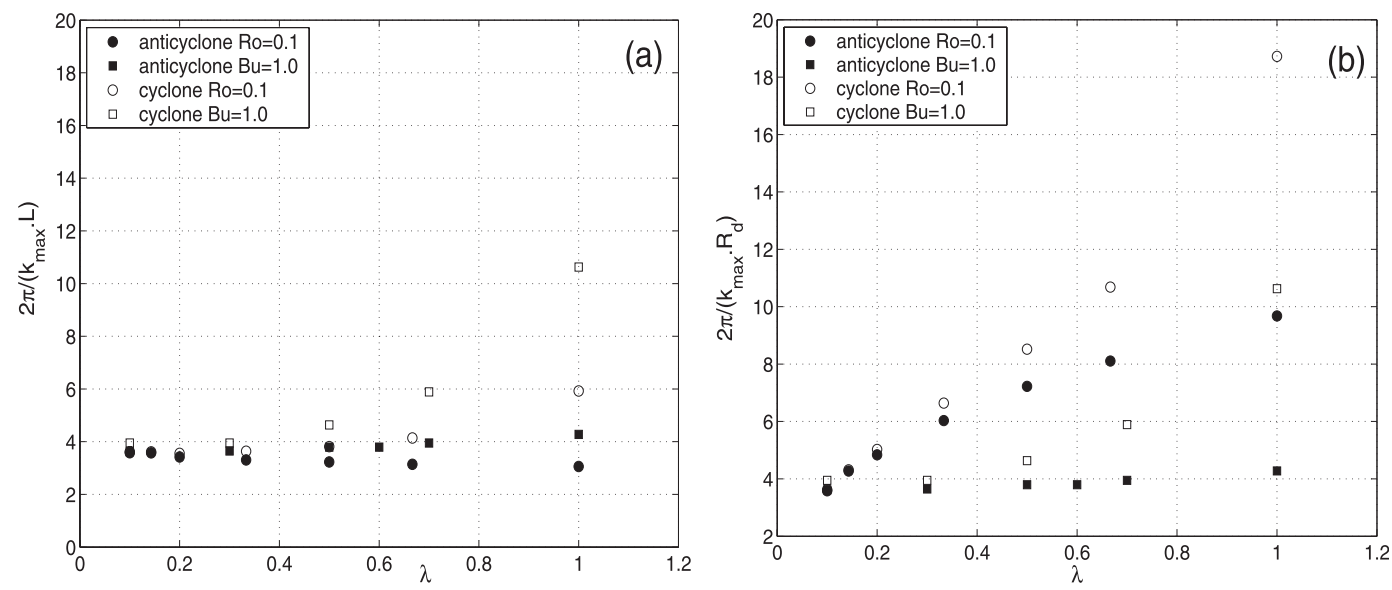

FIG. 5. Evolution of the most unstable wavelength normalized by (a) the shear width $L$ and (b) the deformation radius $R_{d}$ for the anticyclonic (filled symbols) and the cyclonic (open symbols) shear as a function of the surface deviation $\lambda$. 

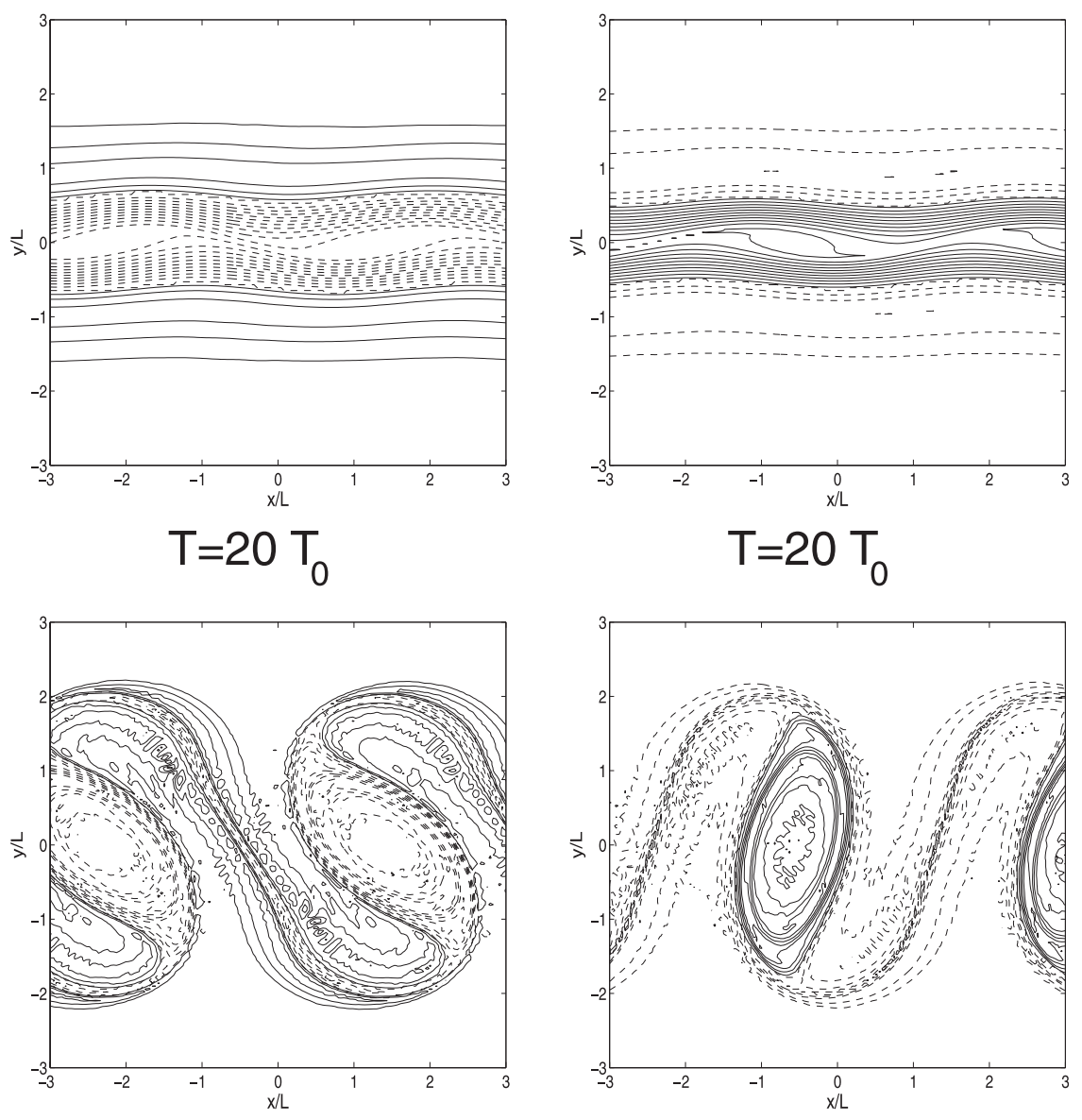

$\mathrm{T}=35 \mathrm{~T}_{0}$
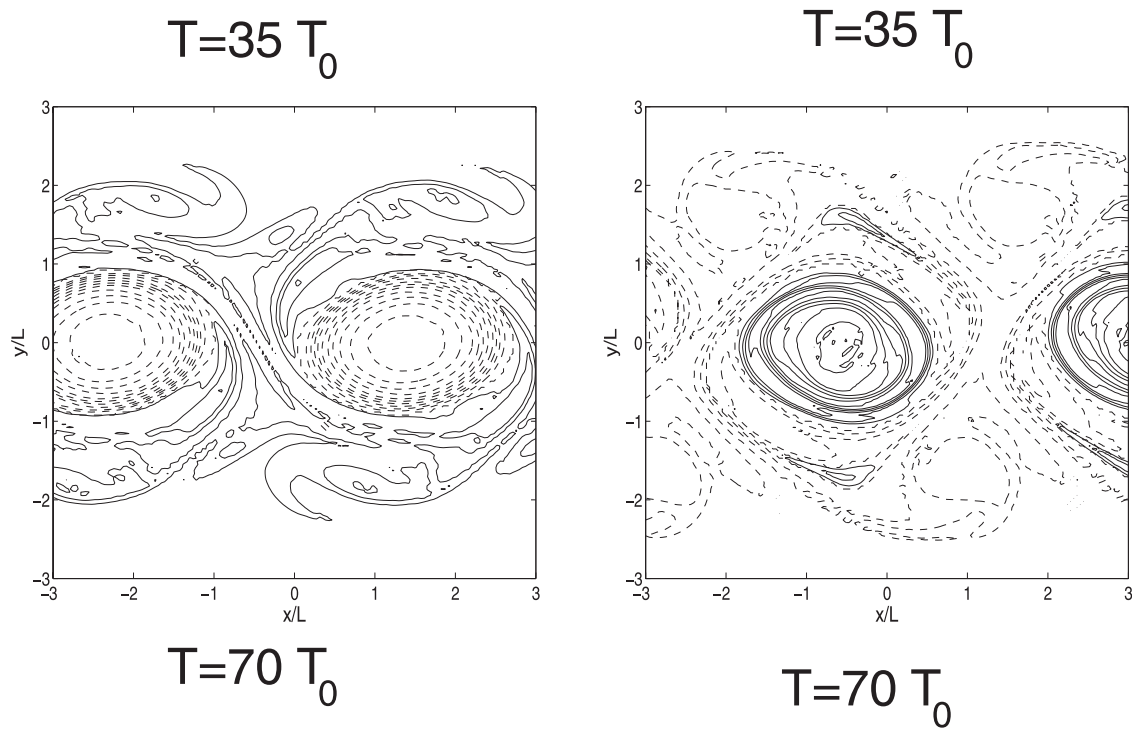

FIG. 6. Nonlinear evolution of PV for the (left) anticyclonic and (right) cyclonic shear at $\mathrm{Bu}=1.0$ and $\mathrm{Ro}=0.1$. The extremum of PV anomaly is $\pm 0.35 f /\left(g h_{0}\right)$ and the contour interval is $3 \cdot 10^{-2} f /\left(g h_{0}\right)$. Note that, for this figure and Figs. 7 and 8 , the whole domain of calculation extends to $y \in[-5 ; 5]$. 

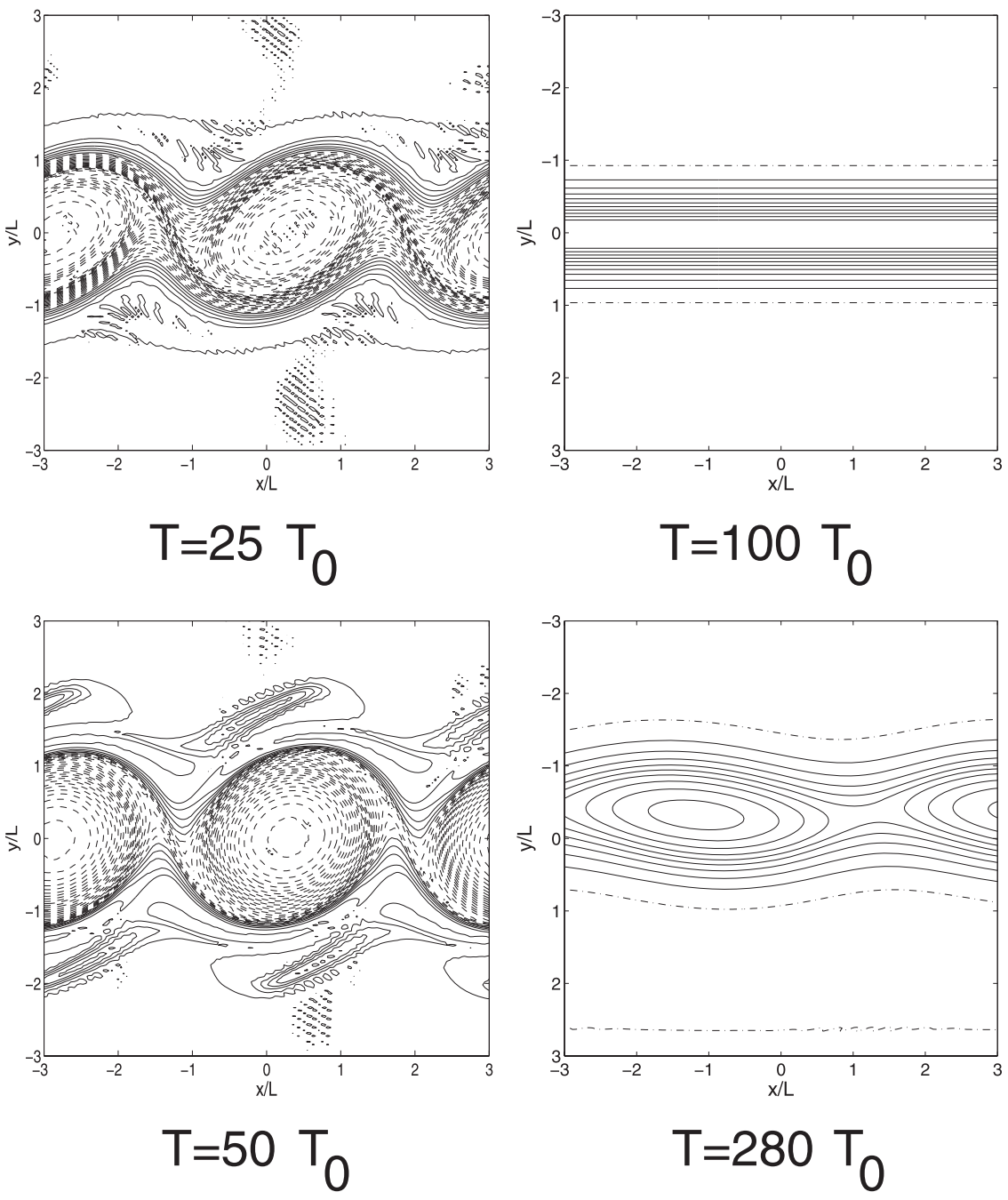

$\mathrm{T}=280 \mathrm{~T}_{0}$
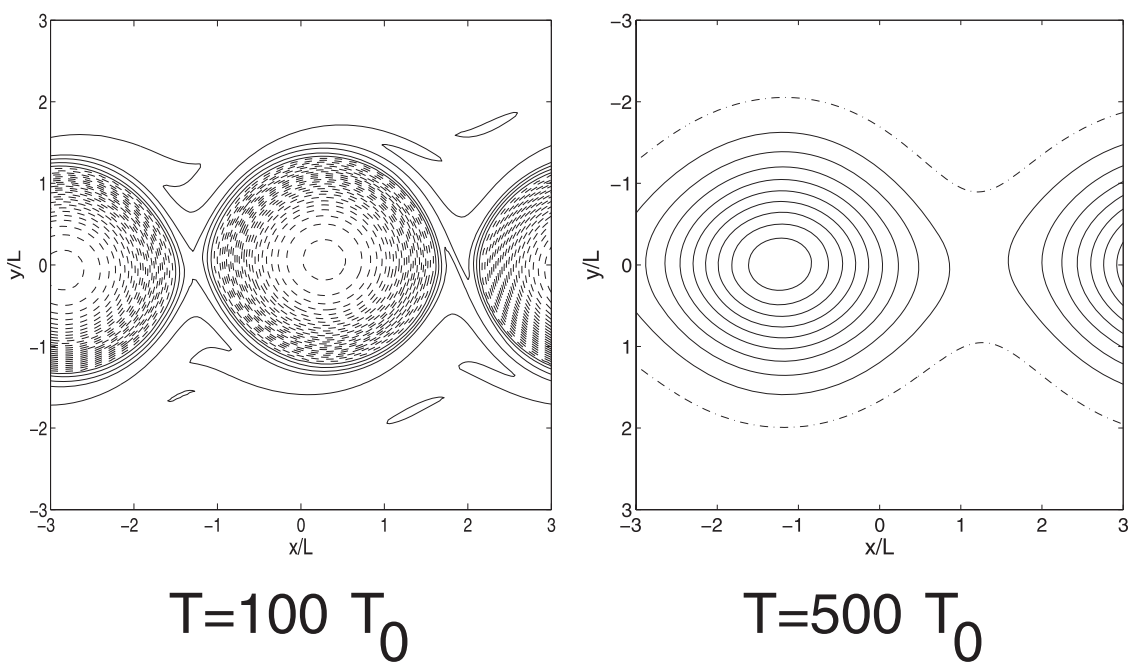

FIG. 7. Nonlinear evolution of PV for the (left) anticyclonic and (right) cyclonic shear at $\mathrm{Bu}=0.1$ and $\mathrm{Ro}=0.1$. The maximum cyclonic PV anomaly is $1.4 f /\left(g h_{0}\right)$ and minimum of anticyclonic PV anomaly is $-0.6 f /\left(g h_{0}\right)$. The contour interval is $6 \times 10^{-2} f /\left(g h_{0}\right)$ for anticyclonic shear and $0.14 f /\left(g h_{0}\right)$ for cyclonic shear. 

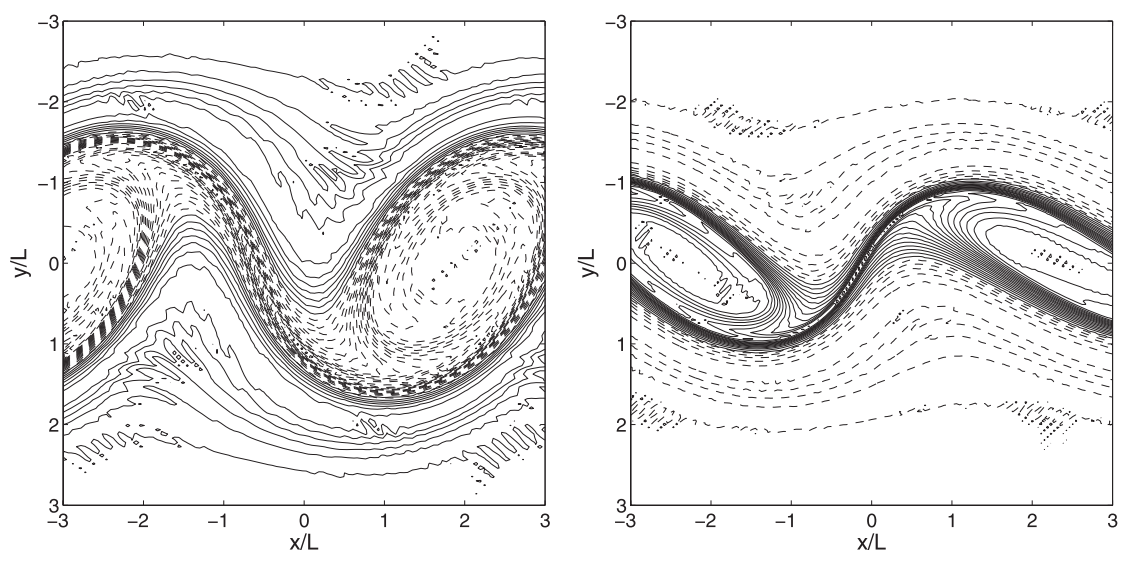

\section{$\mathrm{T}=8 \mathrm{~T}_{0}$}

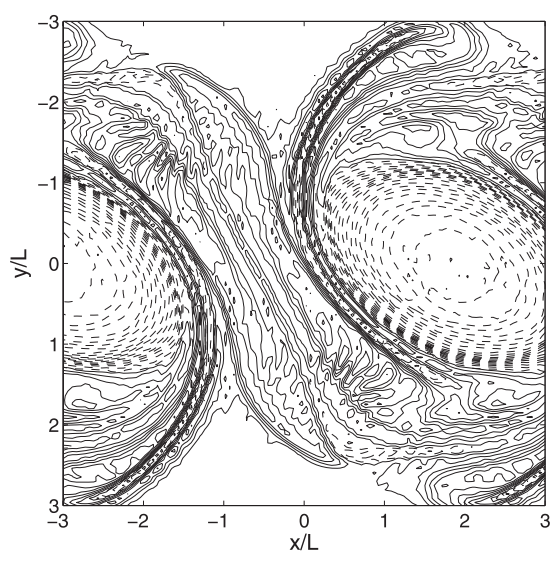

$\mathrm{T}=15 \mathrm{~T}_{0}$

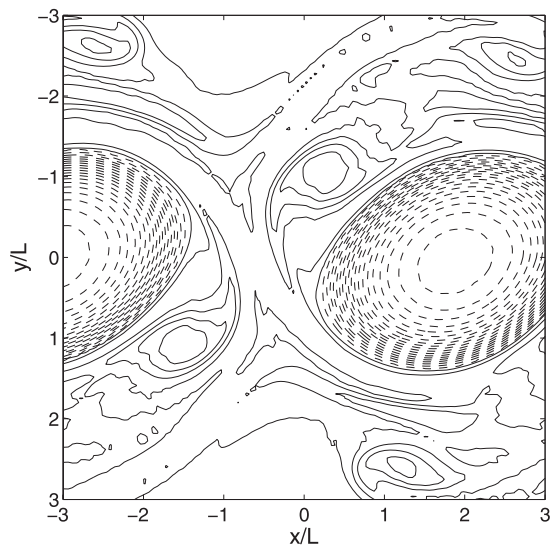

$\mathrm{T}=30 \mathrm{~T}_{0}$
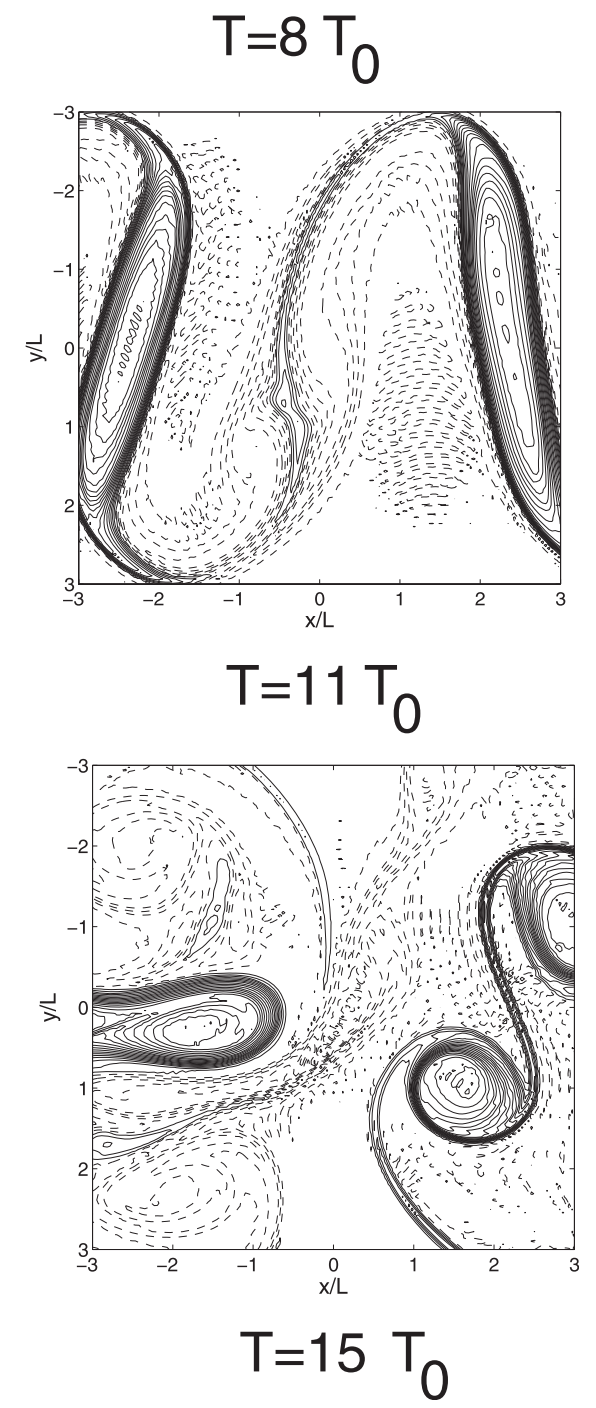

FIG. 8. As in Fig. 7 but at Ro $=0.3$ and $\mathrm{Bu}=1.0$. The maximum cyclonic PV anomaly is $1.6 f /\left(g h_{0}\right)$, and the minimum of anticyclonic PV anomaly is $-0.8 f /\left(g h_{0}\right)$. The contour interval is $4 \times 10^{-2} f /\left(g h_{0}\right)$ for anticyclonic shear and $8 \times 10^{-2} f /\left(g h_{0}\right)$ for cyclonic shear. 

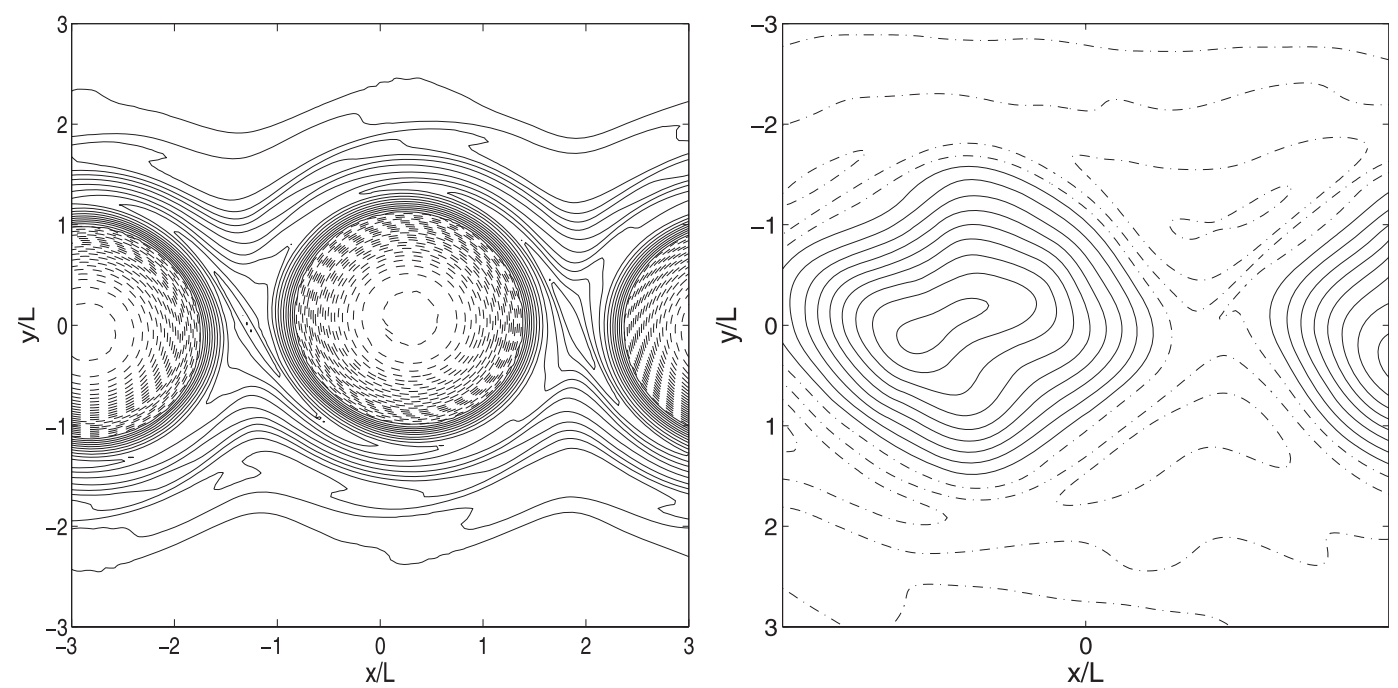

FIG. 9. Vorticity for (left) anticyclonic shear at $T=100 T_{0}$ and (right) cyclonic shear at $T=500 T_{0}$ for $\mathrm{Bu}=0.1$ and Ro $=0.1$. The minimum of anticyclonic vorticity is $-0.3 f$ (the contour interval is 0.03 ) and the maximum of cyclonic vorticity is $0.17 f$ (contour interval is 0.017 ).

\section{Bickley jet}

\section{a. Basic flow}

The barotropic instability of the two-dimensional Bickley jet has been extensively studied (Lipps 1962; Howard and Drazin 1964; Maslowe 1991; Engevik 2004). For large or finite Burger numbers (i.e., twodimensional or QG models) the unstable jet generates cyclonic and anticyclonic eddies symmetric in their size, strength, and shape. For finite Rossby numbers the study of Poulin and Flierl (2003) exhibits a significant cycloneanticyclone asymmetry. To explore the large-scale effect on the jet stability, we study the linear and the nonlinear destabilization of a barotropic jet in the frontal regime (small Rossby number Ro $=0.1$ but finite isopycnal deviation $\lambda=0.5$ ). To allow easier comparisons with previous stability analysis and analytical results, we use the geostrophically balanced Bickley jet (Fig. 10) defined as follows:

$$
\bar{U}(y)=\cosh ^{-2} y+u_{0}
$$

and

$$
\bar{\eta}(y)=-\lambda\left(\tanh y+y u_{0}\right)
$$

where the lateral extent of the domain is $2 y_{b}=20$. Due to geostrophic balance and periodic boundary conditions, the mean velocity must vanish and the central jet is surrounded by a weak reverse flow $u_{0}=-y_{b}^{-1} \tanh y_{b}$. This reverse flow is not needed when using, for instance, free-slip boundary conditions in a finite-width channel
(Poulin and Flierl 2003) and introduces a small difference in the basic flow, compared to previous studies. The velocity, vorticity, surface deviation, and potential vorticity of the Bickley jet for $\lambda=0.1$ and $\lambda=0.5$ are plotted in Fig. 10.

\section{b. Linear stability in $Q G$ and $F G$ regimes}

In Fig. 11 we present the growth rate of unstable modes in the quasigeostrophic regime $\mathrm{Bu}=1.0$ and $\mathrm{Ro}=0.1$. The maximum dimensionless growth rate $\sigma_{\max }=0.062$ is slightly larger than the value $\sigma_{\max }=0.05$ found by Poulin and Flierl. We have checked that this $20 \%$ difference can be explained by the slight difference between the basic flows (not shown). Since our nonlinear simulations are performed with a spectral code enforcing periodic boundary conditions, we present the linear stability analysis of the modified Bickley jet, Eqs. (5) and (6), despite these differences. We have verified that the dependence on the Burger number, discussed below, is insensitive to these details (not shown).

We have also found that the stability curve presents bumps only when the numerical convergence criterion detailed in section $2 \mathrm{~b}(1)$ is not satisfied. For $N=256$ collocation points, a first bump is observed at dimensionless wavenumber $k=1.5$ and a second one at $k=1.8$. Therefore, it seems that such bumps, also present in Poulin and Flierl (2003), are numerical artifacts due to a lack of resolution. In what follows the computations are performed with $N=1024$ collocation points.

In appendix $\mathrm{C}$, we derive analytically the neutral eigenmodes of the QG asymptotic model. This leads to two neutral eigenmodes: a varicose mode at $k=\sqrt{1-\mathrm{Bu}^{-1}}$ 

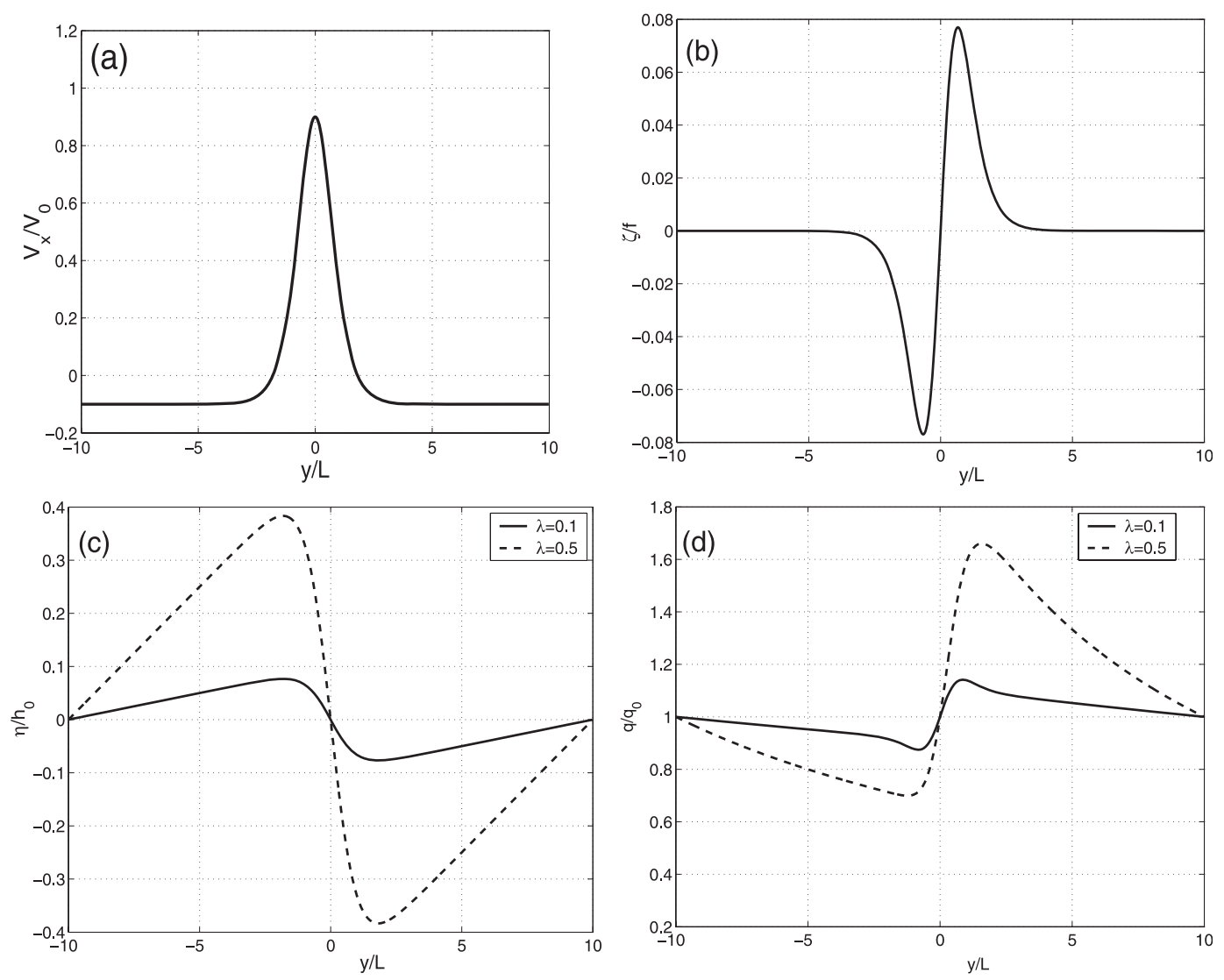

FIG. 10. The Bickley jet basic state (a) velocity, (b) vorticity, (c) relative surface deviation, and (d) PV for $\lambda=0.1$ and $\lambda=0.5$.

and a sinuous mode at $k=\sqrt{4-\mathrm{Bu}^{-1}}$. The varicose branch of instability disappears for $\mathrm{Bu}<1$, whereas the sinuous branch disappears for $\mathrm{Bu}<0.25$. These bounds, valid in the limit of vanishing Rossby number, illustrate the stabilizing effect of a small Burger number $\mathrm{Bu}=\left(R_{d} / L\right)^{2}$, that is, a small deformation radius. For $\mathrm{Bu}=1(\lambda=0.1)$, the neutral mode wavenumber $k R_{d}=1.78$ is very close to the $\mathrm{QG}$ prediction $k R_{d}=\sqrt{4-\mathrm{Bu}^{-1}}=1.73$, despite the finite Rossby number Ro $=0.1$. We expect the varicose branch to disappear near $\lambda=\mathrm{Ro} / \mathrm{Bu}>0.1$ and the Bickley jet to be completely stabilized near $\lambda>0.4$. The actual thresholds differ somewhat in our shallow-water calculations owing to the finite Rossby number and slightly different basic flow.

As for the localized shear layer, we first study the linear stability properties of the flow within the RSW model from the quasigeostrophic regime $(\mathrm{Ro}=0.1, \lambda=$ $0.1)$ to the frontal regime $(\mathrm{Ro}=0.1, \lambda \simeq 1)$. As expected, for the whole parameter range studied we get only one unstable mode, corresponding to the sinuous mode. This normal mode is mainly localized in the center of the jet and despite a small shift of the secondary PV peak the unstable mode keeps the same shape (Fig. 12b).
As expected from the QG analysis, the Bickley jet is strongly stabilized in the RSW model when the frontal regime is reached. The growth rate strongly decreases and the unstable wavelength increases when the surface deviation parameter $\lambda$ becomes finite (Fig. 12a). In the

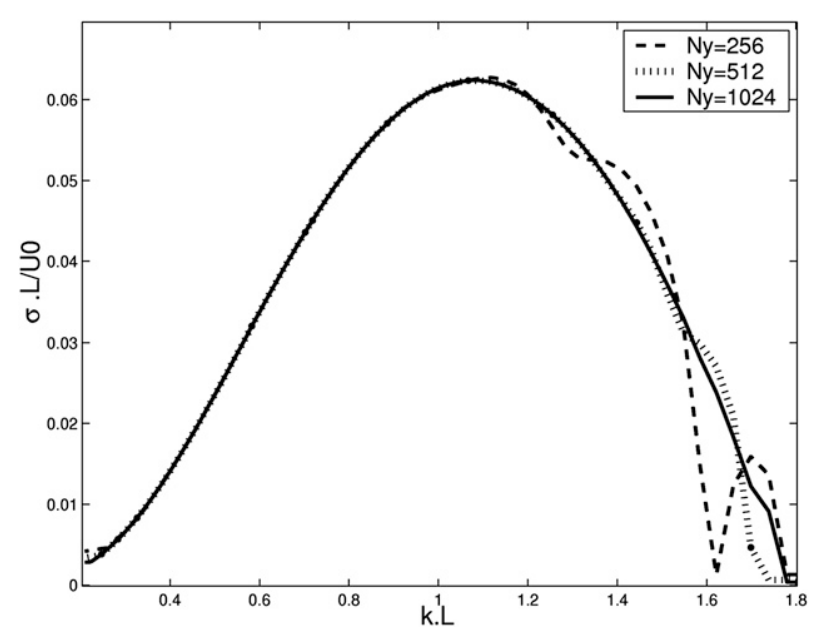

FIG. 11. Growth rate of the Bickley jet at different resolution for $\mathrm{Bu}=1.0$ and $\mathrm{Ro}=0.1$. 

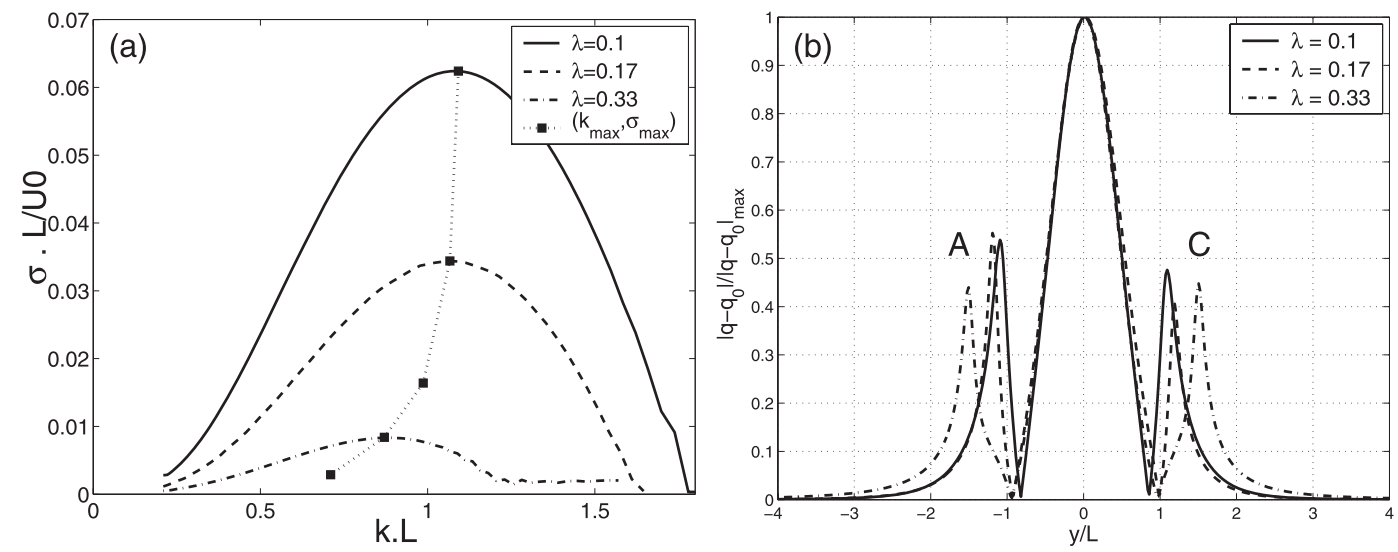

FIG. 12. (a) Growth rate of the Bickley jet for various deviation of the free surface $\lambda$; the Rossby number is fixed at $\mathrm{Ro}=0.1$. The dashed line with square spots indicates $\left(k_{\max }, \sigma_{\max }\right)$ for intermediate values of $\lambda$. (b) PV anomaly of the most unstable mode.

RSW model, the modified Bickley jet is completely stabilized when $\lambda>0.6$.

As far as linear stability is concerned, the Bickley jet is always destabilized by a nearly symmetric sinuous mode. There is no signature of a selective destabilization of anticyclonic vorticity region in the frontal regime.

The evolution of the dimensionless $e$-folding time $1 /\left(\sigma_{\max } T_{0}\right)$ and the dimensionless wavelength $2 \pi /\left(k R_{d}\right)$ as a function of the relative surface deviation $\lambda$ are plotted in Fig. 13. The $e$-folding time of the sinuous mode of the barotropic Bickley jet increases from 20 to 250 days when the relative deviation becomes finite $\lambda=0.5$. We should note here that these values overestimate the observed $e$-folding time of the large-scale oceanic jet. For example, available measurements (Halkin and Rossby 1985 ) indicate that, for the Gulf Stream, Ro $=0.1-0.2$ and $\mathrm{Bu}=0.1$, which is close to the parameters we consider. However, the Gulf Stream exhibits a short $e$-folding time between 3 and 6 days (Watts and Johns 1982; Kontoyiannis and Watts 1994) and a longer one between about 12 and 25 days (Lee and Cornillon 1996). This discrepancy may be due to the barotropic limitation of the RSW model. The baroclinicity of oceanic jets has a strong impact on their stability. In a wide range of parameters, Baey et al. (1999) show that the baroclinic instability can be more efficient to create vortices than the barotropic instability. Hence, if the frontal regime stabilizes the barotropic jets, we could expect that the stability of large-scale oceanic jets are controlled only by the baroclinic processes.

\section{c. Nonlinear evolution}

As for the localized shear flow, the nonlinear evolution of the jet instability and the vortex formation are
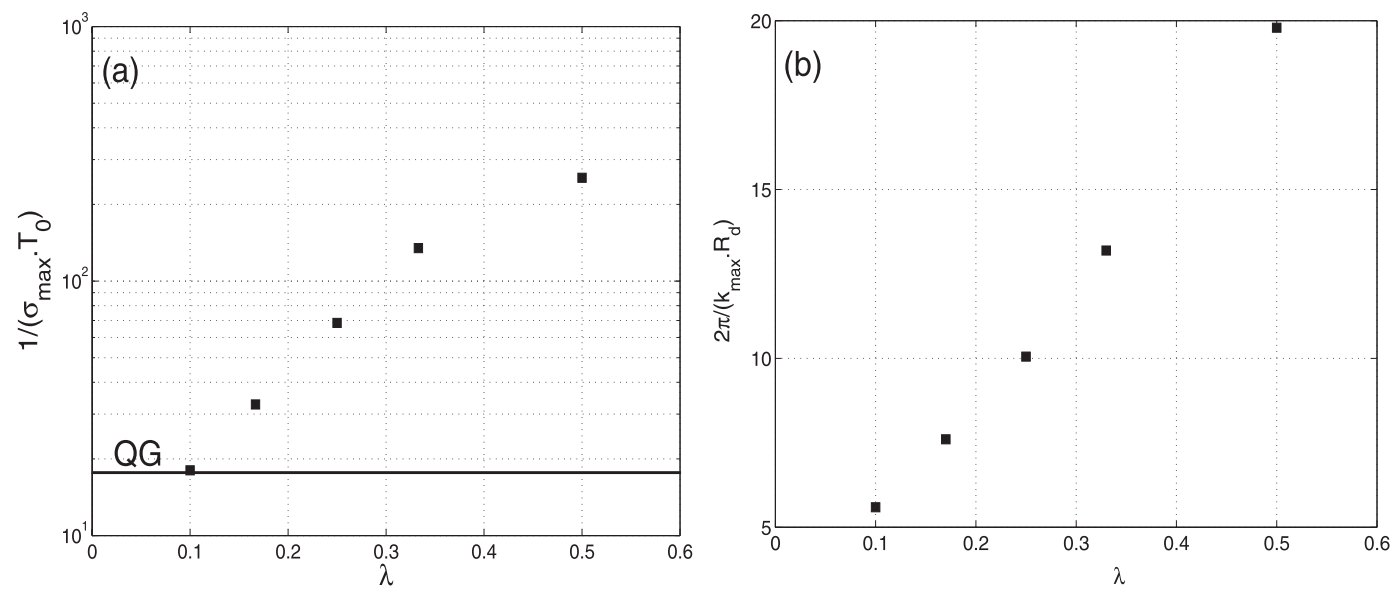

FIG. 13. Semilog plot of (a) the dimensionless $e$-folding time and (b) the dimensionless wavelength for the Bickley jet as a function of the relative surface deviation $\lambda$. 
studied by direct numerical simulations. The dynamical evolution of the unstable Bickley jet is computed in the quasigeostrophic regime (Fig. 14, left) and in the frontal regime (Fig. 14, right). As expected, for the quasigeostrophic regime $(\lambda=0.1)$, the nonlinear saturation of the sinuous mode does not induce a substantial cycloneanticyclone asymmetry. Vortices of both signs are formed with very similar size, shape, and amplitude. However, for finite isopycnal deviation $(\lambda=0.5)$ once coherent vortices are formed, the cyclonic eddies tend to be stretched in an elongated boomerang shape, while the large-scale anticyclones remain coherent and almost circular. A similar behavior was found by Poulin and Flierl (2003) when the Rossby number reaches finite values. In this cyclogeostrophic regime the surface deviation becomes finite as in the frontal regime. Hence, even if the linear growth of unstable sinuous mode does not lead to a strong cyclone-anticyclone asymmetry, the nonlinear evolution of the system does.

\section{Parallel wake flows}

\section{a. Basic flow}

Unlike the Bickley jet, parallel wake flows may exhibit a significant cyclone-anticyclone asymmetry at the linear stage of the instability. According to both numerical (Perret et al. 2006a; Dong et al. 2007) and laboratory studies (Perret et al. 2006b), when the deformation radius is smaller than the typical width of the wake (i.e., in the frontal regime), the wake flow tends to have the same stability properties as two independent shear layers. The most unstable mode is localized in the anticyclonic shear, and the convectively unstable flow behaves as a noise amplifier (Perret et al. 2006a). The main goal of this section is to quantify the linear or the nonlinear coupling mechanism between the two opposite shears of a wake flow. Hence, in the following stability analysis, we construct various parallel wake flows as a combination of two localized shear flows having the same amplitude. The typical width of the wake $D$ is defined as the distance between the two shears, that is, the distance between the two vorticity extrema. For an oceanic or a laboratory wake, the typical width $D$ corresponds to the mean island diameter or the cylinder diameter. As in section 3, the extent of each localized shear is $L$, and we introduce the dimensionless wake parameter $\delta=D / L$. The parallel wake flow is divided in three regions (Figs. 15a,b): two lateral shears separated by a central region of extent $d=D-L$ having a constant velocity $U / V_{0}=-1$. Hence, we study a family of wake flow profiles having various widths $\delta$ according to
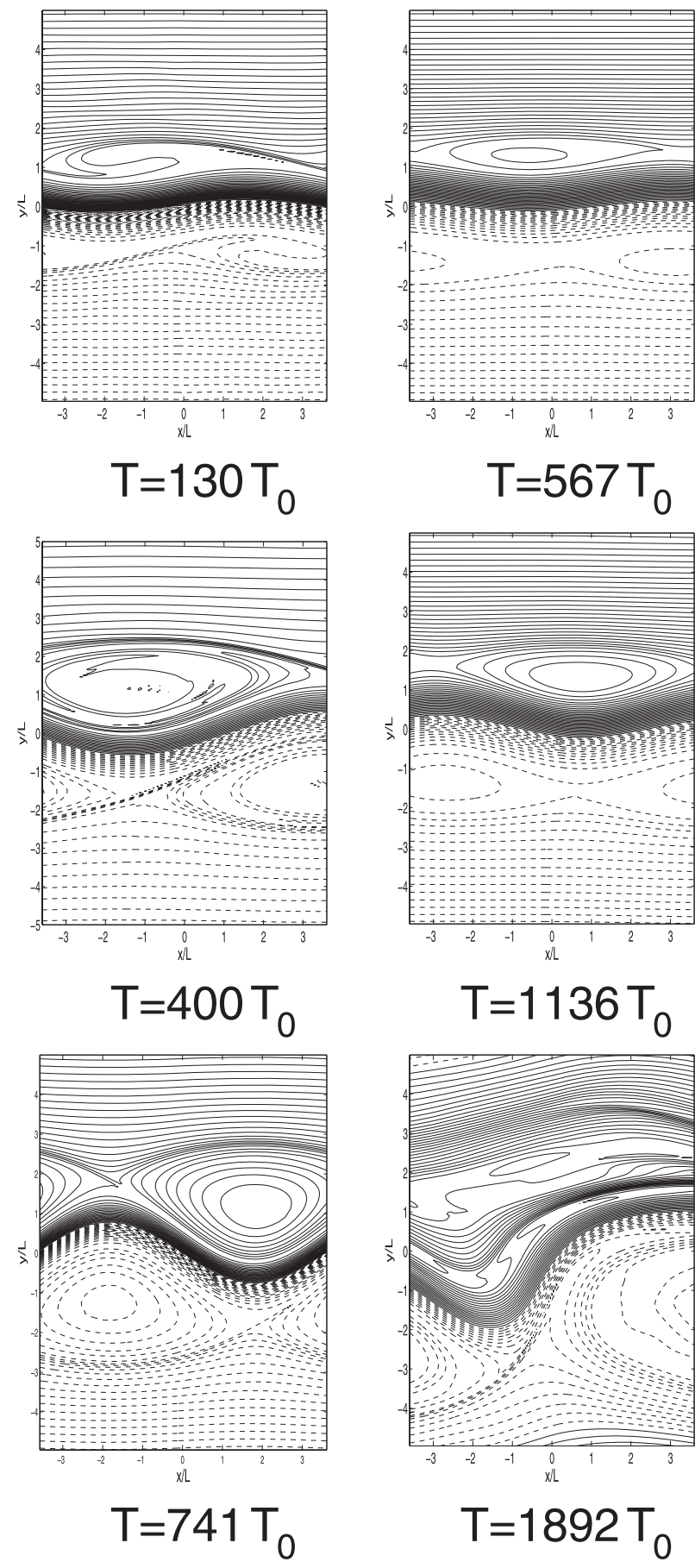

FIG. 14. Nonlinear evolution of PV for the Bickley jet at (left) $\lambda=0.1$ and (right) $\lambda=0.5$ and Ro fixed at 0.1. The maximum and minimum PV anomaly is $\pm 0.15 f /\left(g h_{0}\right)$ for $\lambda=0.1$ and $\pm 0.5 f /\left(g h_{0}\right)$ for $\lambda=0.5$. The contour interval is $5 \times 10^{-3} f /\left(g h_{0}\right)$ for $\lambda=0.1$ and $2 \times 10^{-2} f /\left(g h_{0}\right)$ for $\lambda=0.5$. 

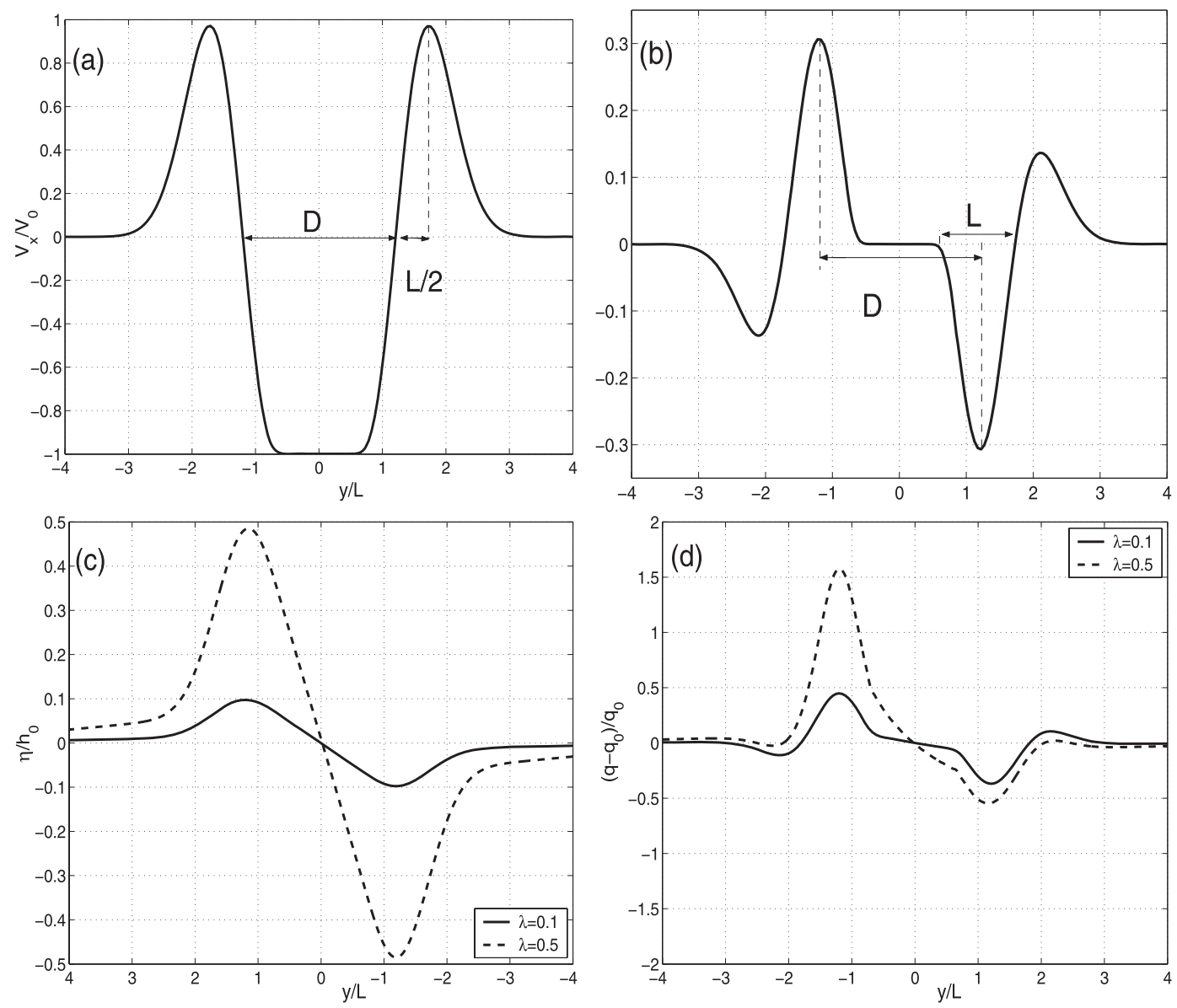

FIG. 15. (a) Velocity profile and (b) vorticity profile for a parallel wake flow of typical width $\delta=2.5$. The corresponding (c) relative surface deviation and (d) PV are plotted for $\lambda=0.1$ (solid line) and $\lambda=0.5$ (dashed line).

$$
\begin{aligned}
& y \leq-\frac{\delta-1}{2}: \bar{U}(y)=-c(y+\delta / 2) e^{-2(y+\delta / 2)^{2}}, \\
&|y| \leq \frac{\delta-1}{2}: \quad \bar{U}(y)=-1,
\end{aligned}
$$

and

$$
y \geq \frac{\delta-1}{2}: \quad \bar{U}(y)=c(y-\delta / 2) e^{-2(y-\delta / 2)^{2}},
$$

where $c=2 e^{1 / 2}$ is a normalization coefficient. The relative surface deviation of this parallel wake flow family is derived from $\bar{U}(y)$ according to the geostrophic balance condition

$$
\left(\bar{U}(y)=-\frac{1}{\lambda} \frac{d \bar{\eta}}{d y}\right)
$$

The typical variations of the relative surface deviation and the potential vorticity, for a wake profile corresponding to $\delta=2.5$, are plotted in Fig. 15 for the quasigeostrophic regime $(\lambda=0.1)$ and the frontal regime $(\lambda=0.5)$.

\section{b. Linear stability in the $Q G, F G$, and cyclogeostrophic regimes}

The linear stability of a parallel wake profile corresponding to $\delta=2.5$ is studied in the quasigeostrophic regime, in the frontal regime (various Burger numbers while the Rossby number is kept small at Ro $=0.1$ ), and in the cyclogeostrophic regime (various Rossby numbers while the Burger number is kept constant at $\mathrm{Bu}=1)$. The eigenmode, called mode A (mode C), corresponds to an unstable perturbation preferentially localized in the anticyclonic (cyclonic) shear region of the wake profile as shown in Fig. 16. However, in the quasigeostrophic regime $(\mathrm{Bu}=1$ corresponding to $\lambda=0.1)$, each eigenmode (A or C) extends spatially in both shears (Fig. 16b). In this regime, the two most unstable modes have roughly the same growth rates: $\sigma_{a} L / V_{0} \simeq 0.52$ and $\sigma_{c} L / V_{0} \simeq 0.45$ (Fig. 16a). These values are very close to the growth rates 

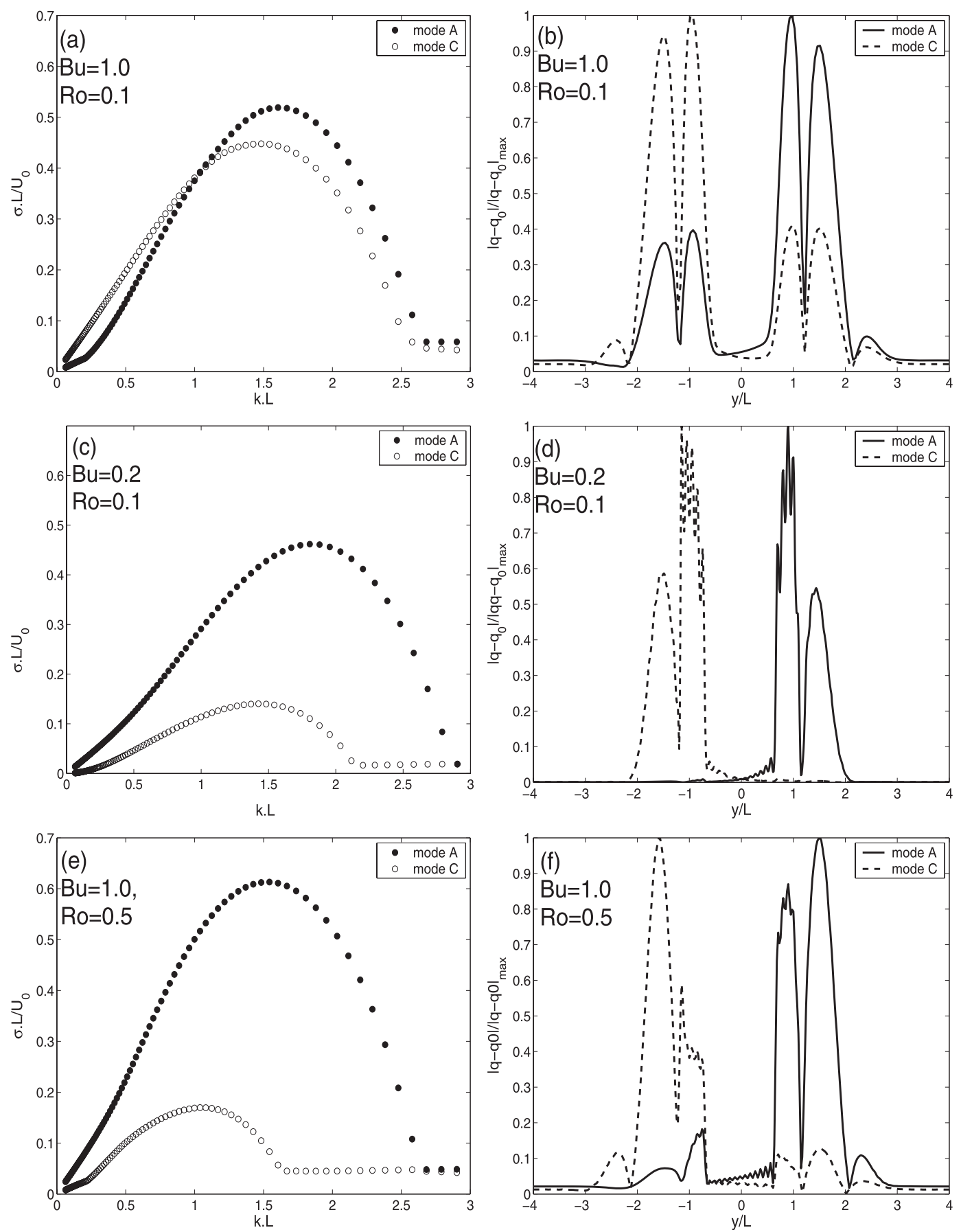

FIG. 16. The two most unstable branches growth rate of wake flow for $\delta=2.5$ in (a) QG regime, (c) frontal regime, and (e) cyclogeostrophic regime and PV anomaly of the most unstable modes in (b) QG, (d) FG, and (f) cyclogeostrophic regimes.

of the anticyclonic and the cyclonic localized shear layers found in section 3 (Fig. 2). The differences between the growth rates $\sigma_{a}$ and $\sigma_{c}$ are probably due to small ageostrophic effects induced by the small but nevertheless finite $\mathrm{Ro}=0.1$. Hence, unstable perturbations will grow exponentially at the same rate on both sides of the wake.
Whereas, for a frontal regime $(\mathrm{Bu}=0.2)$, corresponding to significant surface deviation $\lambda=0.5$, the most unstable eigenmode (mode A) is strictly localized in the anticyclonic shear region (Fig. 16d), and the mode $\mathrm{C}$ has a reduced growth rate $\sigma_{c} L / V_{0} \simeq 0.14$ (Fig. 16c). For the cyclogeostrophic regime $\mathrm{Bu}=1$ and $\mathrm{Ro}=0.5$ the growth 
rate of the unstable mode $\mathrm{A}$, localized in the anticyclonic shear, remains constant $\sigma_{a} L / V_{0} \simeq 0.6$ while the growth rate of the cyclonic mode $\mathrm{C}$ is, here again, strongly reduced $\sigma_{c} L / V_{0} \simeq 0.16$ (Fig. 16e). Hence, in both cases, the unstable perturbations will grow much faster on the anticyclonic side of the wake. We recover here the results previously found in the frontal regime (Perret et al. 2006a) and extend them to the cyclogeostrophic case: beyond the quasigeostrophic regime the linear stability of wake flow may induce a selective destabilization of regions with anticyclonic vorticity.

If we reduce the central region $(d \rightarrow 0$ corresponding to $\delta \rightarrow 1$ ), the parallel wake flow becomes similar to a parallel jet flow. In this case, as for the Bickley jet, we expect the most unstable eigenmode to be a sinuous mode and therefore the cyclone-anticyclone asymmetry should be strongly weakened at the linear stage of the instability. Hence, to study the influence of the width parameter on the wake flow stability, we compare the previous cases where $\delta=2.5$ with the case where $\delta=1.22$ (Fig. 17). As expected, Fig. 17b shows for the quasigeostrophic regime $(\lambda=0.1)$ that the most unstable branch corresponds to a symmetric mode (i.e., a sinuous perturbation) and the second branch to an antisymmetric mode (i.e., a varicose perturbation). In this regime, the maximum sinuous growth rate $\sigma_{s} L / V_{0} \simeq 0.6$ is significantly higher than the maximum varicose growth rate $\sigma_{v} L / V_{0} \simeq$ 0.35. Hence, like the Bickley jet, the most unstable mode corresponds to a symmetric perturbation even if the growth rate of the antisymmetric perturbation (varicose mode) is not negligible. When the wake enters the frontal regime $(\lambda=0.5$ corresponding to $\mathrm{Bu}=0.2$ and $\mathrm{Ro}=0.1)$, the symmetry properties of the two most unstable eigenmodes change. The unstable modes corresponding to the sinuous branch (varicose branch) remain almost symmetric (antisymmetric) in the center of the wake, but not on the border of the jet. The amplitude of the most unstable modes of the sinuous branch are slightly amplified on the anticyclonic side of the wake, while the unstable modes of the varicose branch tend to be slightly amplified on the cyclonic side of the wake. When the wake enters the cyclogeostrophic regime $(\lambda=0.5$ corresponding to $\mathrm{Bu}=1$ and $\mathrm{Ro}=0.5)$, the most unstable mode remains symmetric (sinuous mode) and both the cyclonic and the anticyclonic shears are destabilized.

Therefore, at the linear stage of the wake instability a cyclone-anticyclone asymmetry starts to appear in the frontal or the cyclogeostrophic regime, but the amplitude of the asymmetry depends both on the Rossby number and the relative width of the wake $\delta$. When the width of the wake is large $(\delta=2.5)$, the cyclonic and anticyclonic shears of the wake are less connected and for smaller deformation radius (frontal regime) or finite
Rossby numbers the most unstable perturbation is fully localized within the anticyclonic shear (Fig. 16d or Fig. $16 f)$. For smaller width $(\delta=1.22)$, the unstable perturbations of large-scale $(\mathrm{Bu} \ll 1)$ or cyclogeostrophic $(\mathrm{Ro} \simeq 1)$ wake flows may destabilize almost equally both sides of the wake (Figs. 17d,f). Hence, the cycloneanticyclone selection of the unstable modes at the linear stage of the instability depends on both $\mathrm{Bu}$ and Ro, and the width parameter of the wake $\delta$.

\section{c. Coupling of the cyclonic and anticyclonic shears}

To quantify more accurately the coupling between the unstable modes on the anticyclonic and the cyclonic sides of the wake, we introduce the correlation coefficient $R$,

$$
R\left(q_{1}, q_{2}\right)=\frac{\operatorname{Re}\left(\int q_{\text {mode } 1}^{*} q_{\text {mode } 2} d y\right)}{\sqrt{\int\left|q_{\text {mode } 1}\right|^{2} d y \int\left|q_{\text {mode } 2}\right|^{2} d y}},
$$

where $q^{*}$ indicates the complex conjugate, Re the real part, and $q_{1}(y)$ and $q_{2}(y)$ are the spatial distribution of the two most unstable eigenmodes PV. When $R=0$, the two modes are uncorrelated spatially, whereas for $R=1$ the two modes are spatially identical. If $R>0.2$ (this threshold is arbitrary), we say that the two eigenmodes are coupled, whereas if $R<0.2$ we say that they are uncoupled. Moreover, we define the parameter $\gamma \equiv d / R_{d}=$ $(\delta-1) / \mathrm{Bu}^{1 / 2}$ to quantify the relative extent of the central zone of the wake separating the two shears on both sides of the wake. According to Fig. 18, in the geostrophic regime $(\mathrm{Ro}=0.1)$, the boundary between the coupled and the uncoupled modes depends mainly on $\gamma$. At the first order of approximation, the critical line $\gamma_{c}=2.5$ defines reasonably well the separation between the coupled and the uncoupled eigenmodes for a wide range of Burger numbers. However, this critical value may slightly decrease for a finite Rossby number; indeed, for Ro $=0.5$ and $\mathrm{Bu}=1$ we have found $\gamma_{c}=1.8$. Hence, when the two shear layers of the wake profile are separated by more than two or three deformation radii, the unstable eigenmodes tend to be uncoupled at the linear stage of the instability. In such case, we can use the results of the first section on each localized shear flows, indicating that the anticyclonic shear of the wake is the most unstable when the isopycnal displacement become finite.

\section{d. Nonlinear evolution}

The nonlinear evolution of the instability of parallel wake flows and the vortex formation are studied by direct numerical simulations (Figs. 19 and 20). In agreement with our previous results on the stability of parallel wake flows, the nonlinear interactions between vortices of opposite sign enhance the cyclone-anticyclone 

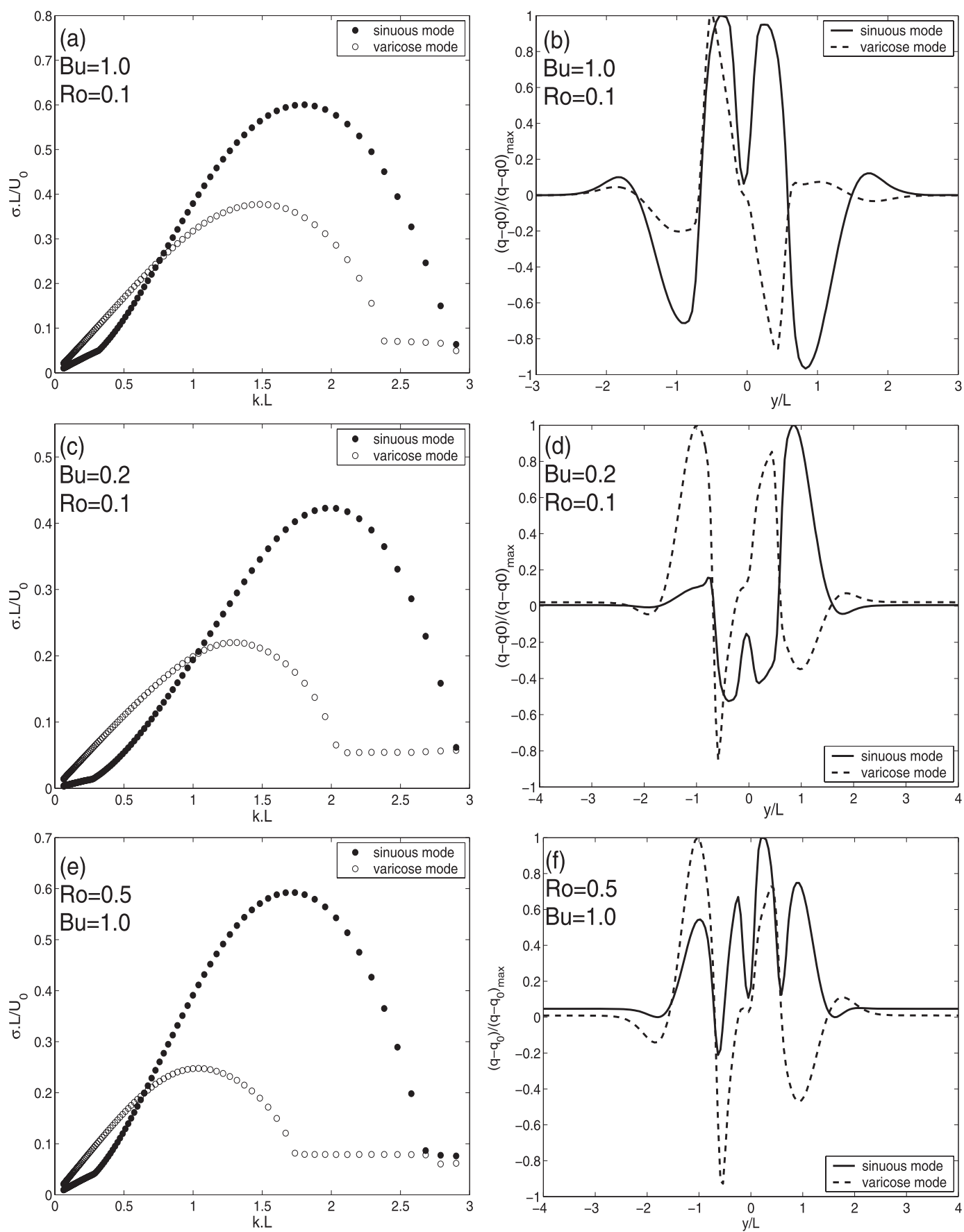

FIG. 17. The two most unstable branches growth rate of wake flow for $\delta=1.22$ in (a) QG regime, (c) frontal regime, and (e) cyclogeostrophic regime and PV anomaly of the most unstable modes in (b) QG, (d) FG, and (f) cyclogeostrophic regimes.

asymmetry of large-scale wakes (Perret et al. 2006a). Cyclonic vortices are stretched or strongly deformed into triangular shapes in comparison with the anticyclonic vortices (Fig. 19), which remain robust and circular in the frontal regime $(\mathrm{Bu}=0.2$ and $\mathrm{Ro}=0.1)$. For a small width parameter $(\delta=1.22)$ the first meanders lead to the formation of vortices of both signs with a slight cyclone-anticyclone asymmetry (Fig. 20, top middle), then the nonlinear interactions between vortices lead to a strong distortion of cyclonic structures (Fig. 20, bottom middle). Moreover, for both quasigeostrophic and frontal regimes, the opposite sign vortices are aligned 


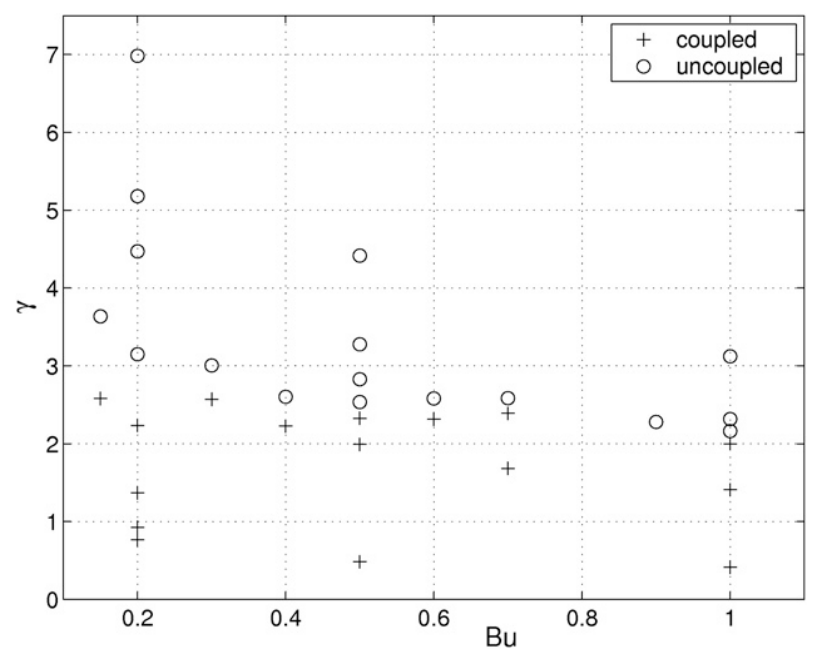

FIG. 18. Coupling of the two most unstable modes in the $\mathrm{Bu}-\gamma$ parameter space for geostrophically balanced flows $(\mathrm{Ro}=0.1)$.

along the same line, unlike the standard Karman vortex street. For a larger width parameter $(\delta=2.5)$ the first meander leads to a significant cyclone-anticyclone asymmetry in the vortex formation (Fig. 19, top middle). This is the signature of a selective destabilization of the anticyclonic shear region of the wake at the linear stage of the instability. Indeed, for this case $(\delta=2.5, \gamma \simeq 3.3)$, according to Figs. 16 and 18, the perturbations growing in each shear layer are not coupled. Coherent vortices emerge first on the anticyclonic side of the wake and, once they are formed, the nonlinear interactions with the cyclonic shear lead to the formation of distorted cyclones.

\section{Conclusions}

The stability of various parallel flows was investigated in the context of the RSW model. This simple model describes the barotropic surface motion in the upper thermocline and neglects the baroclinic interactions of the surface and the deep oceanic flow. However, this model captures the non-QG dynamics and the fast inertia-gravity wave motion. Several aspects of the cyclone-anticyclone asymmetry of large-scale and parallel flows (shear, jets, and wakes) were then studied.

According to the various stability analysis performed on a wide variety of parallel flows, we emphasize that the barotropic instability of oceanic shears, jets, and wake flows favors the formation of large-scale anticyclonic eddies. In both the frontal regime (small Rossby number and finite isopycnal displacements) and the cyclogeostrophic regime (finite Rossby number) an anticyclonic shear flow will have higher growth rates than a cyclonic one. The linear stage of the instability induces a strong cyclone-anticyclone asymmetry and favors the development of unstable perturbations in the anticyclonic shear. The nonlinear saturation leads to the formation of coherent and almost axisymmetric anticyclones, whereas the cyclones tend to be more elongated in the shear direction once they are formed. Other studies on the stability of isolated eddies have shown that, beyond the QG regime, anticyclones tend to be more stable and coherent than their cyclonic counterparts (Arai and Yamagata 1994; Baey and Carton 2002; Stegner and Dritschel 2000; Graves et al. 2006). Hence, the opposite stability properties of anticyclonic shear regions (unstable) and anticyclonic eddies (stable) may help to contribute to the predominance of large-scale and long-lived anticyclones in the oceans.

The second result of this paper is to confirm that the cyclone-anticyclone asymmetry of parallel flows may occur at the linear stage or at the nonlinear stage of the instability, respectively. Two different mechanisms are then involved. Indeed, unlike a localized shear flow, the stability analysis of a Bickley jet, in the frontal regime, reveals no distinction between regions of positive (cyclonic) or negative (anticyclonic) vorticity. The most unstable perturbation corresponds here to a sinuous mode, leading to the meandering of the whole jet. However, when the nonlinear saturation occurs and coherent structures are formed, large-scale anticyclones tend to be axisymmetric, whereas the cyclonic structures are highly distorted and elongated along the jet meander. We recover here the patterns of non-QG turbulent flows where several coherent structures interact together with a predominance of large-scale (larger than the deformation radius) and robust anticyclones (Arai and Yamagata 1994; Polvani et al. 1994; Linden et al. 1995). The nonlinear mechanism involved here could be the strong sensitivity of cyclonic structures to external strain perturbations studied by Graves et al. (2006). The Bickley jet favors the emergence of robust anticyclonic eddies only at the nonlinear stage.

Moreover, we demonstrate how the coupling between opposite shears may suppress, at the linear stage of the instability, the cyclone-anticyclone asymmetry. Assuming that the deformation radius is a characteristic length scale of interaction between localized structures, two localized shear flows will not "feel" each other if they are distant enough. We found that, if the distance $D-L=$ $\gamma R_{d}$ separating two shear regions is larger than two or three deformation radii, the two localized shears will be linearly uncoupled. In such case, if the widths $L$ of the shears are large enough, the flow will exhibit a significant cyclone-anticyclone asymmetry. Hence, for large-scale wakes the linear perturbations will grow much faster in the anticyclonic vorticity region. However, if the two shear regions are too close to each other $(\gamma \lesssim 2)$, the opposite shears will be coupled at the linear stage and the 

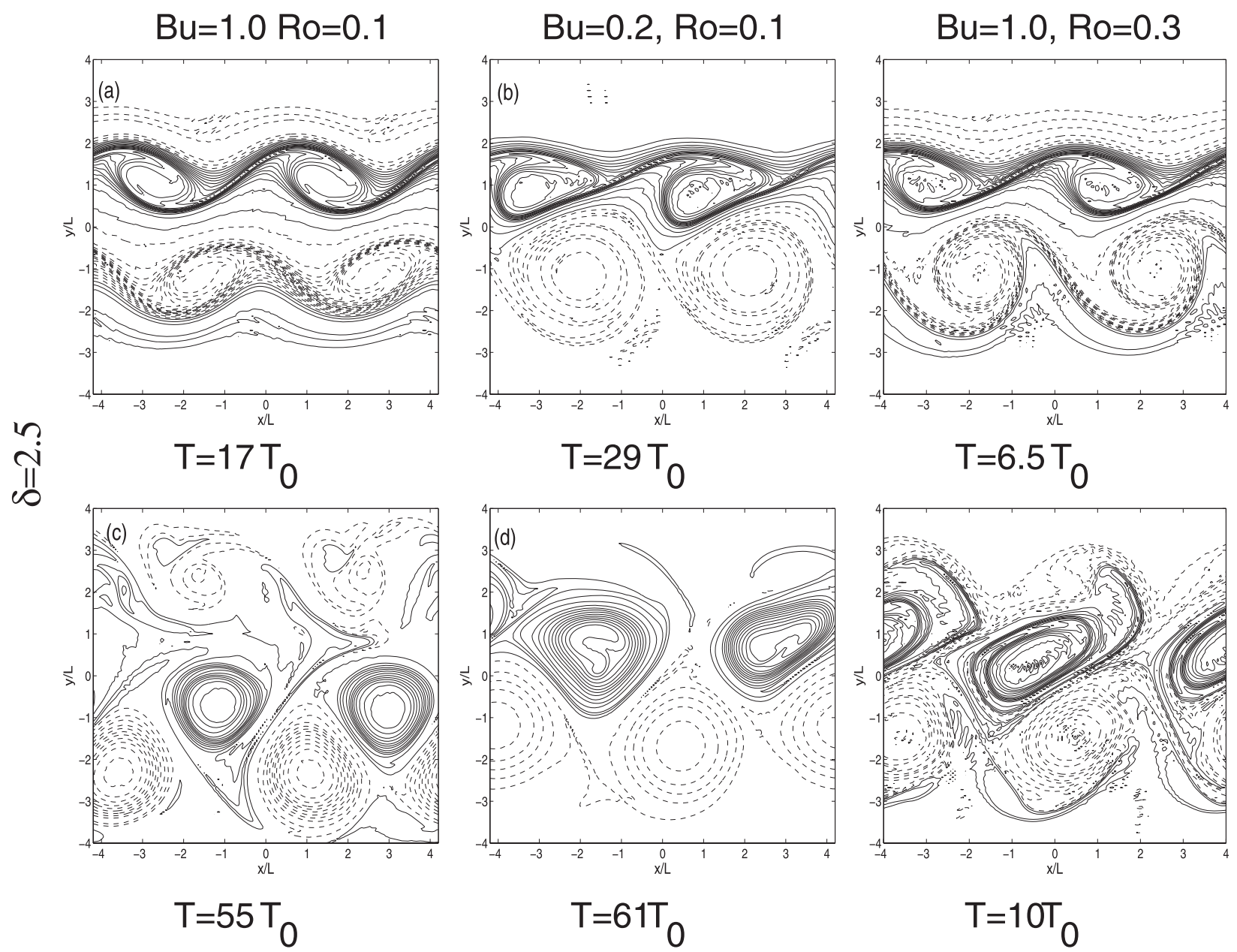

FIG. 19. Nonlinear evolution of PV field for a wake profile corresponding to $\delta=2.5$ (left) in QG regime, (middle) in frontal regime, and (right) in cyclogeostrophic regime. The range of PV is $\pm 0.5 f /\left(g h_{0}\right)$ in QG regime, $\pm 1.6 f /\left(g h_{0}\right)$ in frontal regime, and $\pm 1.9 f /\left(g h_{0}\right)$ in cyclogeostrophic regime. The contour intervals are $3.0 \times 10^{-3} f /\left(g h_{0}\right)$ in QG regime, $0.11 f /\left(g h_{0}\right)$ in frontal regime, and $0.13 f /\left(g h_{0}\right)$ in cyclogeostrophic regime.

most unstable perturbation will then be a sinuous mode. In such case, no distinction occurs between the cyclonic or the anticyclonic vorticity region in the linear stage of instability, as for the Bickley jet.

Even if the baroclinic instability is not taken into account, this study may contribute to a better understanding of the preferred formation of large-scale anticyclones. In the frontal regime (small Rossby number and finite isopycnal displacements) or the cyclogeostrophic regime (finite Rossby number) only large-scale anticyclones will emerge in a reasonable time if the barotropic shear instability is the dominant mechanism of eddy formation. For realistic large-scale wakes, such as the Hawaiian or the Canaria archipelago wakes, the eddy formation is mainly governed by the barotropic shear instability. However, for these specific cases, both largescale anticyclonic shear (width larger than the deformation radius) and smaller cyclonic shear coexist. The large-scale anticyclonic shear will be in FG regime, whereas the cyclonic shear will obey the QG or the cyclogeostrophic regime. In such conditions both shears are unstable with similar growth rates, the anticyclonic shear in the FG regime being slightly less (more) unstable than the cyclonic shear in the QG regime (cyclogeostrophic). Thus, eddies of both signs, but different size, will be formed. The cyclonic eddies should then be smaller and form before the anticyclonic ones. Therefore, a more detailed study, taking into account the interactions and the relative stability between shears of different width and intensity, is needed to provide quantitative predictions on typical vortex shedding frequencies.

Acknowledgments. We gratefully acknowledge JeanMarc Chomaz for enlightening discussion. 


\section{$\mathrm{Bu}=1.0 \mathrm{Ro}=0.1$}

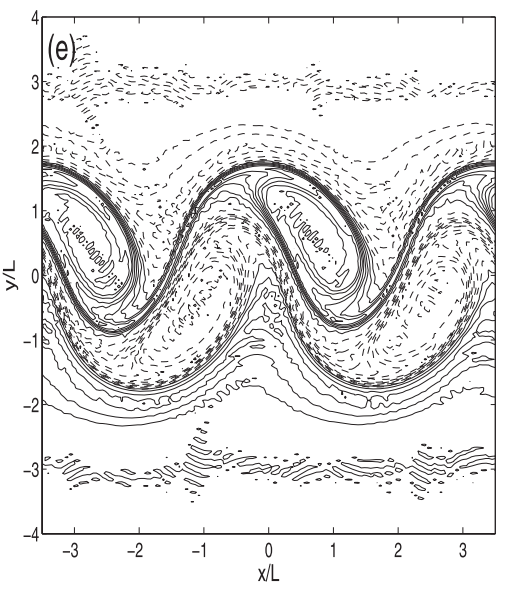

$\underset{\text { กิ }}{\stackrel{\text { II }}{\pi}}$

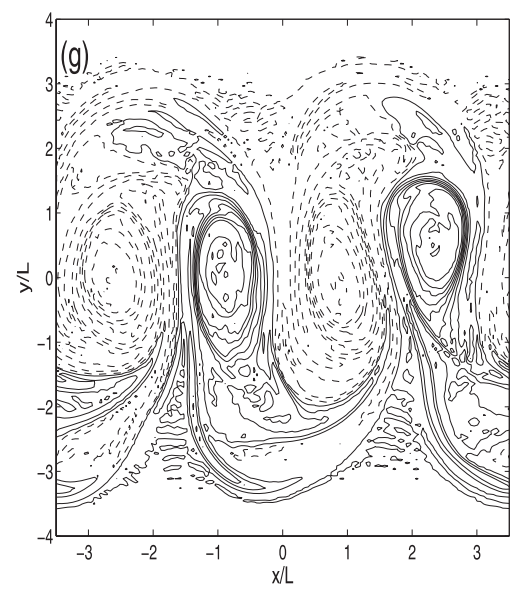

$\mathrm{T}=44 \mathrm{~T}$
$\mathrm{Bu}=0.2, \mathrm{Ro}=0.1$

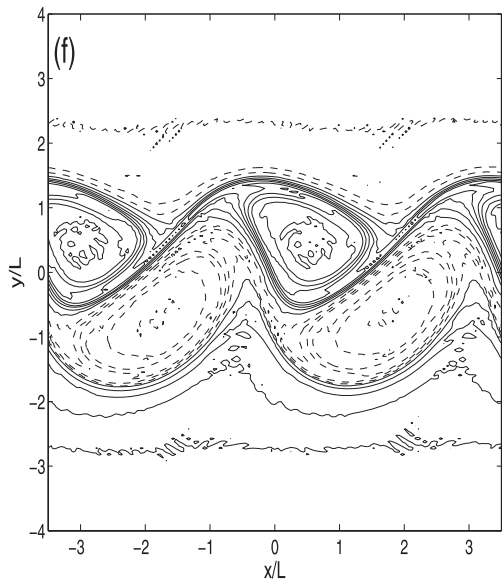

$\mathrm{T}=25 \mathrm{~T}_{0}$

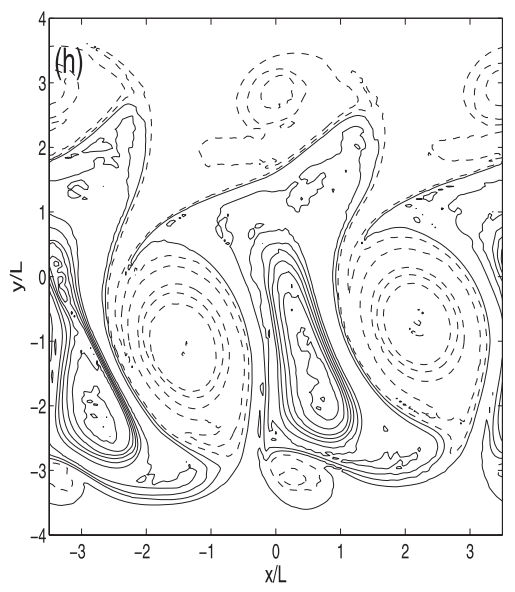

$\mathrm{T}=49 \mathrm{~T}$
$\mathrm{Bu}=1.0, \mathrm{Ro}=0.3$

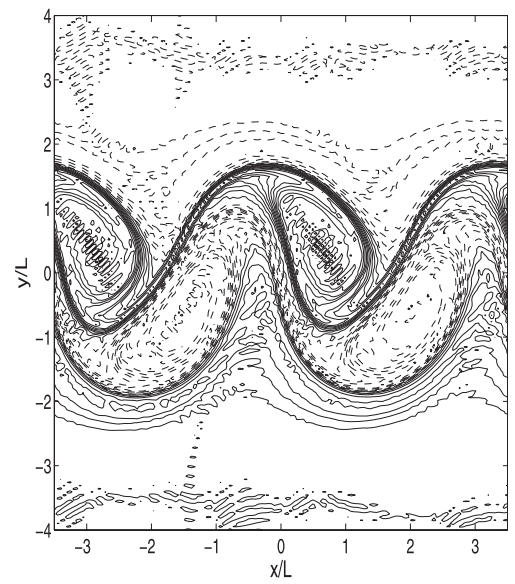

$\mathrm{T}=6 \mathrm{~T}_{0}$

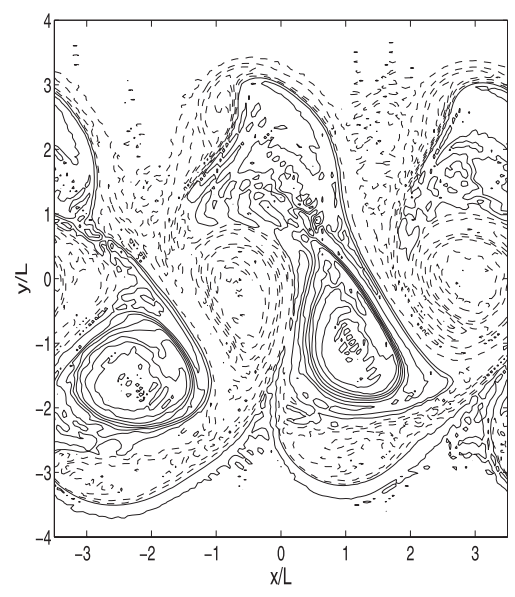

$\mathrm{T}=10 \mathrm{~T}$

FIG. 20. As in Fig. 19 but corresponding to $\delta=1.22$. The range of PV is $\pm 0.5 f /\left(g h_{0}\right)$ in QG and frontal regimes and $\pm 1.5 f /\left(g h_{0}\right)$ in cyclogeostrophic regime. The contour intervals are $3.0 \times 10^{-2} f /\left(g h_{0}\right)$ in QG and frontal regimes and $0.16 f /\left(g h_{0}\right)$ in cyclogeostrophic regime.

\section{APPENDIX A}

\section{Rayleigh Criterion for the Frontal Geostrophic Model}

Here we extend the classical Rayleigh criterion for instability of parallel incompressible two-dimensional flows to the frontal geostrophic model. The dimensionless frontal model is written as

$$
\frac{\partial \eta}{\partial t}-J\left(\eta,(1+\eta) \Delta \eta+\frac{1}{2}(\nabla \eta)^{2}\right)=0,
$$

where $\eta$ is the surface deviation and $J(a, b)=\partial_{x} a \partial_{y} b-$ $\partial_{y} a \partial_{x} b$ is the Jacobian operator (Cushman-Roisin 1986).
Any parallel flow $\eta_{0}(y)$ is a stationary solution of Eq. (A1). To study the stability of that flow, we decompose the surface deviation $\eta$ as follows:

$$
\eta=\eta_{0}(y)+\tilde{\eta}(x, y, t)
$$

where $\tilde{\eta}$ is a small perturbation. The linearized frontal equation is then

$$
\begin{aligned}
& \frac{\partial \tilde{\eta}}{\partial t}-\left(1+\eta_{0}\right)\left[J\left(\eta_{0}, \Delta \tilde{\eta}\right)+J\left(\tilde{\eta}, \Delta \eta_{0}\right)\right] \\
& \quad-J\left(\eta_{0}, \nabla \eta_{0} \cdot \nabla \tilde{\eta}\right)-\frac{1}{2} J\left(\tilde{\eta},\left(\nabla \eta_{0}\right)^{2}\right)=0 .
\end{aligned}
$$


The perturbation $\tilde{\eta}$ may be decomposed into normal modes,

$$
\tilde{\eta}(x, y, t)=\hat{\eta}(y) e^{i(k x-\omega t)},
$$

where $k$ is prescribed and $\omega$ and $\hat{\eta}(y)$ are complex unknowns. The solution has to be bounded as $x \rightarrow \infty$, implying that $k$ is real. Introducing (A3) into (A2) yields

$$
\begin{gathered}
\omega \hat{\eta}+k\left(1+\eta_{0}\right)\left[\frac{d \eta_{0}}{d y}\left(k^{2} \hat{\eta}-\frac{d^{2} \hat{\eta}}{d y^{2}}\right)+\hat{\eta} \frac{d^{3} \eta_{0}}{d y^{3}}\right] \\
-k\left[\left(\frac{d \eta_{0}}{d y}\right)^{2} \frac{d \hat{\eta}}{d y}+\hat{\eta} \frac{d^{2} \eta_{0}}{d y^{2}} \frac{d \eta_{0}}{d y}\right]=0 .
\end{gathered}
$$

Setting the phase velocity $c=\omega / k=c_{r}+i c_{i}$, one can write

$$
\begin{aligned}
(1 & \left.+\eta_{0}\right) \frac{d \eta_{0}}{d y} \frac{d^{2} \hat{\eta}}{d y^{2}}+\left(\frac{d \eta_{0}}{d y}\right)^{2} \frac{d \hat{\eta}}{d y} \\
- & -\left[c+\left(1+\eta_{0}\right)\left(\frac{d \eta_{0}}{d y} k^{2}+\frac{d^{3} \eta_{0}}{d y^{3}}\right)-\frac{d^{2} \eta_{0}}{d y^{2}} \frac{d \eta_{0}}{d y}\right] \hat{\eta}=0 .
\end{aligned}
$$

Assuming that the basic state profile is monotonic, $d \eta_{0} /$ $d y \neq 0$, then one can divide the previous equation by $d \eta_{0} / d y$,

$$
\begin{aligned}
& \left(1+\eta_{0}\right) \frac{d^{2} \hat{\eta}}{d y^{2}}+\frac{d \eta_{0}}{d y} \frac{d \hat{\eta}}{d y}-\left[\frac{c}{d \eta_{0} / d y}+\left(1+\eta_{0}\right)\left(k^{2}+\frac{d^{3} \eta_{0} / d y^{3}}{d \eta_{0} / d y}\right)-\frac{d^{2} \eta_{0}}{d y^{2}}\right] \hat{\eta} \\
& =\frac{d}{d y}\left[\left(1+\eta_{0}\right) \frac{d \hat{\eta}}{d y}\right]-\left[\frac{c}{d \eta_{0} / d y}+\left(1+\eta_{0}\right)\left(k^{2}+\frac{d^{3} \eta_{0} / d y^{3}}{d \eta_{0} / d y}\right)-\frac{d^{2} \eta_{0}}{d y^{2}}\right] \hat{\eta}=0
\end{aligned}
$$

Assuming, moreover, that $\hat{\eta}$ is a localized perturbation, then $\int_{-\infty}^{+\infty}|\hat{\eta}|^{2} d y$ exists and $\lim _{y \rightarrow \infty}|\hat{\eta}|=0$. Multiplying the equation by the conjugate of the perturbation $\hat{\eta}^{*}$ and integrating between $-\infty$ and $\infty$, we get

$$
\int_{-\infty}^{+\infty} \hat{\eta}^{*} \frac{d}{d y}\left[\left(1+\eta_{0}\right) \frac{d \hat{\eta}}{d y}\right] d y-\int_{-\infty}^{\infty}\left[\frac{c}{d \eta_{0} / d y}+\left(1+\eta_{0}\right)\left(k^{2}+\frac{d^{3} \eta_{0} / d y^{3}}{d \eta_{0} / d y}\right)-\frac{d^{2} \eta_{0}}{d y^{2}}\right]|\hat{\eta}|^{2} d y=0 .
$$

The first term may be integrated by parts:

$$
\begin{aligned}
\int_{-\infty}^{+\infty} \hat{\eta}^{*} \frac{d}{d y}\left[\left(1+\eta_{0}\right) \frac{d \hat{\eta}}{d y}\right] d y= & {\left[\left(1+\eta_{0}\right) \hat{\eta}^{*} \frac{d \hat{\eta}}{d y}\right]_{-\infty}^{+\infty} } \\
& -\int_{-\infty}^{+\infty}\left(1+\eta_{0}\right)\left|\frac{d \hat{\eta}}{d y}\right|^{2} d y
\end{aligned}
$$

The first term on the right-hand side tends to zero at infinity; Eq. (A6) then becomes

$$
\begin{aligned}
& c \int_{-\infty}^{+\infty} \frac{|\hat{\eta}|^{2}}{d \eta_{0} / d y} d y+\int_{-\infty}^{\infty}\left(1+\eta_{0}\right)\left|\frac{d \hat{\eta}}{d y}\right|^{2} d y \\
& \quad+\int_{-\infty}^{\infty}\left[\left(1+\eta_{0}\right)\left(k^{2}+\frac{d^{3} \eta_{0} / d y^{3}}{d \eta_{0} / d y}\right)-\frac{d^{2} \eta_{0}}{d y^{2}}|\hat{\eta}|^{2} d y=0 .\right.
\end{aligned}
$$

There is only one imaginary term in this equation, which must then vanish:

$$
c_{i} \int_{-\infty}^{+\infty} \frac{|\hat{\eta}|^{2}}{d \eta_{0} / d y} d y=0
$$

Therefore, to be unstable $\left(c_{i} \neq 0\right)$, the flow must have a nonmonotonic basic state surface deviation:

$$
\exists y_{0} / \frac{d \eta_{0}}{d y}\left(y_{0}\right)=0
$$

\section{APPENDIX B}

\section{Sensitivity of an Isolated Shear to Details of the Velocity Profile}

The isolated shear defined in section 3 has two lateral vorticity peaks (Fig. B1b). To test the influence of the lateral shears on the stability of a localized shear flow, we compare two parallel flows having the same central shear but different lateral ones (Figs. B1a,b). According to Figs. B1c,d, the growth rate and the wavelength of the most unstable modes are weakly affected by the lateral shears. Hence, if we remain far from an outcropping configuration, the central shear controls the instability of this localized barotropic flow and the results of the following stability analysis can be extended to a large variety of shear flows. 

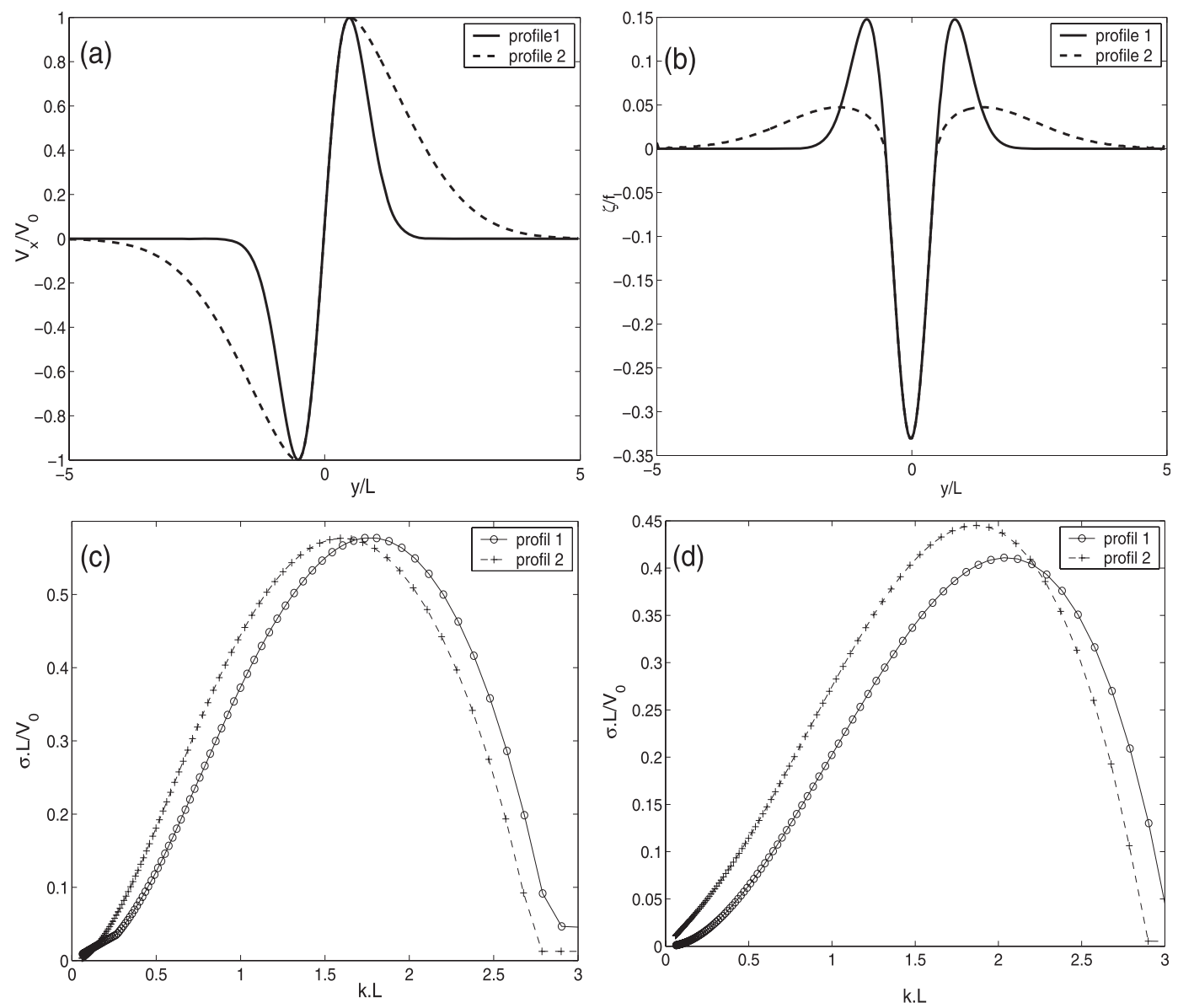

FIG. B1. Comparison between a standard localized shear profile (solid line) and a localized shear flow with less steep lateral shears (dashed line): (a) velocity profile, (b) vorticity profile, and growth rates of the unstable mode for (c) the QG regime $\lambda=0.1$ and (d) the frontal regime $\lambda=1.0$.

\section{APPENDIX C}

\section{Neutral Eigenmodes of the Bickley Jet in a Quasigeostrophic Model}

Any profile $n_{0}(y)$ is a stationary solution of the quasigeostrophic Eq. (3). Inserting $n=n_{0}(y)+\psi(x, y, t)$ into (3) and neglecting terms quadratic in $\psi$ yields the linearized QG equation:

$$
\left(\frac{\partial}{\partial t}+U \frac{\partial}{\partial x}\right)\left(\Delta \psi-\mathrm{Bu}^{-1} \psi\right)-\frac{\partial \psi}{\partial x}\left(U-\mathrm{Bu}^{-1} U\right)=0,
$$

where $U=-d n_{0} / d y$ and $U^{\prime \prime}=d^{2} U / d y^{2}$. Considering a normal mode $\psi(x, y, t)=\phi(y) \exp [i k(x-c t)]$ yields the Rayleigh-Kuo equation,

$$
(U-c)\left[\phi^{\prime \prime}-\left(k^{2}+\mathrm{Bu}^{-1}\right) \phi\right]-\left(U^{\prime \prime}-\mathrm{Bu}^{-1} U\right) \phi=0 .
$$

From now on, $U(y)=\cosh ^{-2} y$ is the Bickley profile. A neutral eigenmode has, by definition, a zero growth rate or equivalently a real phase velocity $c$. Hence, we look for a real phase velocity $c$ and a real wavenumber $k$ such that Eq. (C1) admits a bounded solution $\phi(y)$.

Neutral eigenmodes of the Bickley jet have been found in the context of $\beta$-plane incompressible dynamics (Engevik 2004). In that case, the normal modes satisfy

$$
(U-c)\left(\phi^{\prime \prime}-k^{2} \phi\right)-\left(U^{\prime \prime}-\beta\right) \phi=0 .
$$

Equation (C2) is singular at the abscissa $y_{c}$ of the critical layer such that $U\left(y_{c}\right)=c$ unless $U^{\prime \prime}\left(y_{c}\right)=\beta^{\prime}$; that is, $\beta=$ $(4-6 c) c$. Equation $(\mathrm{C} 2)$ then admits at least the following solutions:

$$
\phi_{1}=e^{-m y}\left(3 \tanh ^{2} y+3 m \tanh y+m^{2}-1\right)
$$

and

$$
\phi_{2}=e^{m y}\left(3 \tanh ^{2} y-3 m \tanh y+m^{2}-1\right)
$$


where $m^{2}=k^{2}+4-6 c$. For these solutions to be bounded when $y \rightarrow \pm \infty$, we need $3-3 m+m^{2}-1=0$; that is, either $m=1$ or $m=2$, in which case $\phi_{1}$ is a multiple of $\phi_{2}$. This leads to two neutral eigenmodes for each value of $c$ (Engevik 2004).

Now Eq. (C1) is of the form (C2) with $\beta=\mathrm{Bu}^{-1} c$. The quasigeostrophic neutral eigenmodes are found using simple substitution into the $\beta$-plane results. Because $\beta=$ $\mathrm{Bu}^{-1} c$, the solutions of $\beta=(4-6 c)$ are $c=0$ (critical layer at infinity) and $c=2 / 3-\mathrm{Bu}^{-1} / 6$. The latter case, the only one we consider here, leads to $m^{2}=k^{2}+\mathrm{Bu}^{-1}$. Finally, $m=1$ and $m=2$ leads to two neutral eigenmodes: one with $k=\sqrt{1-\mathrm{Bu}^{-1}}$ and one with $k=\sqrt{4-\mathrm{Bu}^{-1}}$.

\section{REFERENCES}

Anderson, E., and Coauthors, 1999: LAPACK Users' Guide. 3rd ed. Society for Industrial and Applied Mathematics, $429 \mathrm{pp}$.

Arai, M., and T. Yamagata, 1994: Asymmetric evolution of eddies in rotating shallow water. Chaos, 4, 163-175.

Baey, J. M., and X. Carton, 2002: Vortex multipoles in two-layer rotating shallow-water flows. J. Fluid Mech., 460, 151-175.

— P. Rivière, and X. Carton, 1999: Oceanic jet instability: A model comparison. ESAIM: Proc., 7, 12-23.

Cushman-Roisin, B., 1986: Frontal geostrophic dynamics. J. Phys. Oceanogr., 16, 132-143.

- , and B. Tang, 1990: Geostrophic turbulence and emergence of eddies beyond the radius of deformation. J. Phys. Oceanogr., 20, 97-113.

Dong, C., J. C. McWilliams, and A. F. Shchepetkin, 2007: Island wakes in deep water. J. Phys. Oceanogr., 37, 962-981.

Drazin, P., and W. H. Reid, 1981: Hydrodynamic Stability. Cambridge University Press, 525 pp.

Engevik, L., 2004: A note on the barotropic instability of the Bickley jet. J. Fluid Mech., 499, 315-326.

Flament, P., R. Lumpkin, J. Tournadre, and L. Arni, 2001: Vortex pairing in an unstable anticyclonic shear flow: Discrete subharmonics of one pendulum day. J. Fluid Mech., 440, 401-409.

Graves, L. P., J. C. McWilliams, and M. T. Montgomery, 2006: Vortex evolution due to straining: A mechanism for dominance of strong, interior anticyclones. Geophys. Astrophys. Fluid Dyn., 100, 151-183.

Halkin, D., and T. Rossby, 1985: The structure and transport of the Gulf Stream at $73^{\circ}$. J. Phys. Oceanogr., 15, 1439-1452.

Howard, L., and P. G. Drazin, 1964: On stability of parallel flow of inviscid fluid in a rotating system with variable Coriolis parameter. J. Math. Phys., 43, 83-99.

Johnson, J., 1963: Stability of shearing motion in rotating fluid. J. Fluid Mech., 17, 337-352.

Kontoyiannis, H., and D. R. Watts, 1994: Observations on the variability of the Gulf Stream path between $74^{\circ} \mathrm{W}$ and $70^{\circ} \mathrm{W}$. J. Phys. Oceanogr., 24, 1999-2013.

Lee, T., and P. Cornillon, 1996: Propagation and growth of the Gulf Stream meanders between $75^{\circ}$ and $45^{\circ} \mathrm{W}$. J. Phys. Oceanogr., 26, 225-241.
Linden, P. F., B. M. Boubnov, and S. B. Dalziel, 1995: Source-sink turbulence in a rotating stratified fluid. J. Fluid Mech., 298, 81-112.

Lipps, F. B., 1962: The barotropic instability of the mean winds in the atmosphere. J. Fluid Mech., 12, 397-407.

Maslowe, S., 1991: Barotropic instability of the Bickley jet. J. Fluid Mech., 229, 417-426.

Matsuura, T., and T. Yamagata, 1982: On the evolution of nonlinear planetary eddies larger than the radius of deformation. J. Phys. Oceanogr., 12, 440-456.

McWilliams, J. C., 1985: Submesoscale, coherent vortices in the ocean. Rev. Geophys., 23, 165-182.

Mitchum, G. T., 1995: The source of 90-day oscillations at Wake Island. J. Geophys. Res., 100, 2459-2475.

Nycander, J., and G. G. Sutyrin, 1992: Stationary translating anticyclones on the beta-plane. Dyn. Atmos. Oceans, 16, 473-498.

Olson, D. B., 1991: Rings in the ocean. Annu. Rev. Planet. Sci., 19, 283-311.

_, and R. J. Evans, 1986: Rings of the Agulhas. Deep-Sea Res., 33, 42 .

Pedlosky, J., 1987: Geophysical Fluid Dynamics. 2nd ed. SpringerVerlag, $710 \mathrm{pp}$.

Perret, G., A. Stegner, T. Dubos, J. M. Chomaz, and M. Farge, 2006a: Stability of parallel wake flows in quasigeostrophic and frontal regime. Phys. Fluids, 18, 126602, doi:10.1063/1.2397563.

,,-- M. Farge, and T. Pichon, 2006b: Cyclone-anticyclone asymmetry of large-scale wakes in the laboratory. Phys. Fluids, 18, 036603, doi:10.1063/1.2179387.

Polvani, L. M., J. C. McWilliams, M. A. Spall, and R. Ford, 1994: The coherent structures of shallow-water turbulence: Deformationradius effects, cyclone/anticyclone asymmetry and gravity-wave generation. Chaos, 4, 177-186.

Potylitsin, P. G., and W. R. Peltier, 1999: Three dimensional destabilization of Stuart vortices: The influence of rotation and ellipticity. J. Fluid Mech., 387, 205-226.

Poulin, F. J., and G. R. Flierl, 2003: The nonlinear evolution of barotropically unstable jets. J. Phys. Oceanogr., 33, 2173-2192.

Sangrá, P., and Coauthors, 2005: Life history of an anticyclonic eddy. J. Geophys. Res., 110, C030201, doi:10.1029/2004JC002526.

Stegner, A., and V. Zeitlin, 1995: What can asymptotic expansions tell us about large-scale quasi-geostrophic anticyclonic vortices? Nonlinear Processes Geophys., 2, 186-193.

- and _- 1996: Asymptotic expansions and monopolar solitary Rossby vortices in barotropic and two-layer models. Geophys. Astrophys. Fluid Dyn., 83, 159-194.

— stability of isolated shallow water vortices. J. Phys. Oceanogr., 30, 2562-2573.

_ T. T. Pichon, and M. Beunier, 2005: Elliptical-inertial instability of rotating Karman vortex streets. Phys. Fluids, 17, 066602, doi:10.1063/1.1937348.

Teinturier, S., A. Stegner, S. Viboud, and H. Didelle, 2010: Smallscale instabilities of an island wake flow in a rotating shallowwater layer. Dyn. Atmos. Oceans, 39, 1-24.

Watts, D. R., and W. E. Johns, 1982: Gulf Stream meanders: Observations on propagation and growth. J. Geophys. Res., 87, 9467-9475.

Yanase, S., C. Flores, O. Metais, and J. Riley, 1993: Rotating free-shear flows. I. Linear stability analysis. Phys. Fluids, A5, 2725-2737. 